Article

\title{
Research on Construction Engineering Project Risk Assessment with Some 2-Tuple Linguistic Neutrosophic Hamy Mean Operators
}

\author{
Shengjun $\mathrm{Wu}^{1}{ }^{1}$, Jie Wang ${ }^{1}$, Guiwu Wei ${ }^{1, *(1)}$ and Yu Wei ${ }^{2, *}$ \\ 1 School of Business, Sichuan Normal University, Chengdu 610101, China; wsjn382@sina.com (S.W.); \\ JW970326@163.com (J.W.) \\ 2 School of Finance, Yunnan University of Finance and Economics, Kunming 650221, China \\ * Correspondence: weiguiwu1973@sicnu.edu.cn (G.W.); weiyusy@126.com (Y.W.); \\ Tel.: +86-28-8448-0719 (G.W.)
}

Received: 14 April 2018; Accepted: 4 May 2018; Published: 11 May 2018

\begin{abstract}
In this paper, we expand the Hamy mean (HM) operator, weighted Hamy mean (WHM), dual Hamy mean (DHM) operator, and weighted dual Hamy mean (WDHM) operator with 2-tuple linguistic neutrosophic numbers (2TLNNs) to propose a 2-tuple linguistic neutrosophic Hamy mean (2TLNHM) operator, 2-tuple linguistic neutrosophic weighted Hamy mean (2TLNWHM) operator, 2-tuple linguistic neutrosophic dual Hamy mean (2TLNDHM) operator, and 2-tuple linguistic neutrosophic weighted dual Hamy mean (2TLNWDHM) operator. Then, the multiple attribute decision-making (MADM) methods are proposed with these operators. Finally, we utilize an applicable example in risk assessment for construction engineering projects to prove the proposed methods.
\end{abstract}

Keywords: multiple attribute decision-making (MADM); neutrosophic numbers; 2-tuple linguistic neutrosophic numbers set (2TLNNSs); 2TLNHM operator; 2TLNWHM operator; 2TLNDHM operator; 2TLNWDHM operator; construction engineering projects; risk assessment

\section{Introduction}

Neutrosophic sets (NSs), which were proposed originally by Smarandache [1,2], have attracted the attention of many scholars, and NSs have acted as a workspace in depicting indeterminate and inconsistent information. A NS has more potential power than other modeling mathematical tools, such as fuzzy set [3], intuitionistic fuzzy set (IFS) [4] and interval-valued intuitionistic fuzzy set (IVIFS) [5]. But, it is difficult to apply NSs to solve real life problems. Therefore, Wang et al. [6,7] defined single valued neutrosophic sets (SVNSs) and interval neutrosophic sets (INS), which are characterized by a truth membership, an indeterminacy membership and a falsity membership. Hence, SVNSs and INSs can express much more information than fuzzy sets, IFSs and IVIFSs. Ye [8] proposed a multiple attribute decision-making (MADM) method with correlation coefficients of SVNSs. Broumi and Smarandache [9] defined the correlation coefficients of INSs. Biswas et al. [10] proposed the Technique for Order Preference by Similarity to an Ideal Solution(TOPSIS) method with SVNNs. Liu et al. [11] defined the generalized neutrosophic number Hamacher aggregation for SVNSs. Sahin and Liu [12] defined the maximizing deviation model under a neutrosophic environment. Ye [13] developed some similarity measures of INS. Zhang et al. [14] defined some aggregating operators with INNs. Ye [15] defined a simplified neutrosophic set (SNS). Peng et al. [16] developed aggregation operators under SNS. Peng et al. [17] investigated the outranking approach with SNS, and then Zhang et al. [18] extended Peng's approach. Liu and Liu [19] proposed a power averaging operator 
with SVNNs. Deli and Subas [20] discussed a novel method to rank SVNNs. Peng et al. [21] proposed multi-valued neutrosophic sets. Zhang et al. [22] gave the improved weighted correlation coefficient for interval neutrosophic sets. Chen and Ye [23] proposed Dombi operations for SVNSs. Liu and Wang [24] proposed the MADM method based on a SVN-normalized weighted Bonferroni mean. Wu et al. [25] proposed a cross-entropy and prioritized an aggregation operator with SNSs in MADM problems. Li et al. [26] developed SVNN Heronian mean operators in MADM problems. Zavadskas et al. [27] proposed a model for residential house elements and material selection using the neutrosophic MULTIMOORA method. Zavadskas et al. [28] studied the sustainable market valuation of buildings using the SVN MAMVA method. Bausys and Juodagalviene [29] investigated the garage location selection for residential houses using the WASPAS-SVNS method. Wu et al. [30] proposed some Hamacher aggregation operators under an SVN 2-tuple linguistic environment for MAGDM.

Although SVNS theory has been successfully applied in some areas, the SVNS is also characterized by truth membership degree, indeterminacy membership degree, and falsity membership degree information. However, all the above approaches are unsuitable for describing the truth membership degree, indeterminacy membership degree, and falsity membership degree information of an element of a set by linguistic variables on the basis of the given linguistic term sets, which can reflect a decision maker's confidence level when they are making an evaluation. In order to overcome this limit, we propose the concept of a 2-tuple linguistic neutrosophic numbers set (2TLNNSs) to solve this problem based on SVNS [6,7] and a 2-tuple linguistic information processing model [31]. Thus, how to aggregate these 2-tuple linguistic neutrosophic numbers is an interesting topic. To solve this issue, in this paper, we develop aggregation operators with 2TLNNs based on the traditional operator [32]. In order to do so, the remainder of this paper is set out as follows. In the next section, we propose the concept of 2TLNNSs. In Section 3, we propose Hamy mean (HM) operators with 2TLNNs. In Section 4, we give a numerical example for risk assessment of a construction engineering projects. Section 5 concludes the paper with some remarks.

\section{Preliminaries}

In this section, we propose the concept of using 2-tuple linguistic neutrosophic sets (2TLNSs) based on SVNSs [6,7] and 2-tuple linguistic sets (2TLSs) [31].

\section{1. $2 T L S S$}

Definition 1. Let $S=\left\{s_{i} \mid i=0,1, \ldots, t\right\}$ be a linguistic term set with an odd cardinality. Any label, $s_{i}$, represents a possible value for a linguistic variable, and $S$ can be defined as:

$$
S=\left\{\begin{array}{l}
s_{0}=\text { extremely poor, } s_{1}=\text { very poor }, s_{2}=\text { poor, } s_{3}=\text { medium }, \\
s_{4}=\text { good, } s_{5}=\text { very good }, s_{6}=\text { extremely good } .
\end{array}\right\}
$$

Herrera and Martinez [27,28] developed the 2-tuple fuzzy linguistic representation model based on the concept of symbolic translation. It is used for representing the linguistic assessment information by means of a 2-tuple $\left(s_{i}, \rho_{i}\right)$, where $s_{i}$ is a linguistic label for predefined linguistic term set $S$ and $\rho_{i}$ is the value of symbolic translation, and $\rho_{i} \in[-0.5,0.5)$.

\subsection{SVNSS}

Let $X$ be a space of points (objects) with a generic element in a fixed set, $X$, denoted by $x$. An SVNS, $A$, in $X$ is characterized as the following [6,7]:

$$
A=\left\{\left(x, T_{A}(x), I_{A}(x), F_{A}(x)\right) \mid x \in X\right\}
$$


where the truth membership function, $T_{A}(x)$, indeterminacy-membership, $I_{A}(x)$, and falsity membership function, $F_{A}(x)$, are single subintervals/subsets in the real standard $[0,1]$, that is, $T_{A}(x): X \rightarrow[0,1], I_{A}(x): X \rightarrow[0,1]$ and $F_{A}(x): X \rightarrow[0,1]$. In addition, the sum of $T_{A}(x), I_{A}(x)$ and $F_{A}(x)$ satisfies the condition $0 \leq T_{A}(x)+I_{A}(x)+F_{A}(x) \leq 3$. Then, a simplification of $A$ is denoted by $A=\left\{\left(x, T_{A}(x), I_{A}(x), F_{A}(x)\right) \mid x \in X\right\}$, which is a SVNS.

For a SVNS $\left\{\left(x, T_{A}(x), I_{A}(x), F_{A}(x)\right) \mid x \in X\right\}$, the ordered triple components, $\left(T_{A}(x), I_{A}(x), F_{A}(x)\right)$, are described as a single-valued neutrosophic number (SVNN), and each SVNN can be expressed as $A=\left(T_{A}, I_{A}, F_{A}\right)$, where $T_{A} \in[0,1], I_{A} \in[0,1], F_{A} \in[0,1]$ and $0 \leq T_{A}+I_{A}+F_{A} \leq 3$.

\subsection{TLNSS}

Definition 2. Assume that $\varphi=\left\{\varphi_{0}, \varphi_{1}, \ldots, \varphi_{t}\right\}$ is a 2TLSs with an odd cardinality, $t+1$. If $\varphi=$ $\left\langle\left(s_{T}, \alpha\right),\left(s_{I}, \beta\right),\left(s_{F}, \gamma\right)\right\rangle$ is defined for $\left(s_{T}, \alpha\right),\left(s_{I}, \beta\right),\left(s_{F}, \gamma\right) \in \varphi$ and $\alpha, \beta, \gamma \in[0, t]$, where $\left(s_{T}, \alpha\right),\left(s_{I}, \beta\right)$ and $\left(s_{F}, \gamma\right)$ express independently the truth degree, indeterminacy degree, and falsity degree by $2 T L S s$, then 2TLNSs is defined as follows:

$$
\varphi_{j}=\left\langle\left(s_{T_{j}}, \alpha_{j}\right),\left(s_{I_{j}}, \beta_{j}\right),\left(s_{F_{j}}, \gamma_{j}\right)\right\rangle
$$

where $0 \leq \Delta^{-1}\left(s_{T_{j}}, \alpha_{j}\right) \leq t, 0 \leq \Delta^{-1}\left(s_{I_{j}}, \beta_{j}\right) \leq t, 0 \leq \Delta^{-1}\left(s_{F_{j}}, \gamma_{j}\right) \leq t$, and $0 \leq \Delta^{-1}\left(s_{T_{j}}, \alpha_{j}\right)+$ $\Delta^{-1}\left(s_{I_{j}}, \beta_{j}\right)+\Delta^{-1}\left(s_{F_{j}}, \gamma_{j}\right) \leq 3 t$.

Definition 3. Let $\varphi_{1}=\left\langle\left(s_{T_{1}}, \alpha_{1}\right),\left(s_{I_{1}}, \beta_{1}\right),\left(s_{F_{1}}, \gamma_{1}\right)\right\rangle$ be a 2 TLNN in $\varphi$. Then, the score and accuracy functions of $\varphi_{1}$ are defined as follows:

$$
\begin{gathered}
S\left(\varphi_{1}\right)=\Delta\left\{\frac{\left(2 t+\Delta^{-1}\left(s_{T_{1}}, \alpha_{1}\right)-\Delta^{-1}\left(s_{I_{1}}, \beta_{1}\right)-\Delta^{-1}\left(s_{F_{1}}, \gamma_{1}\right)\right)}{3}\right\}, S\left(\varphi_{1}\right) \in[0, t] \\
H\left(\varphi_{1}\right)=\Delta\left\{\Delta^{-1}\left(s_{T_{1}}, \alpha_{1}\right)-\Delta^{-1}\left(s_{F_{1}}, \gamma_{1}\right)\right\}, H\left(\varphi_{1}\right) \in[-t, t] .
\end{gathered}
$$

Definition 4. Let $\varphi_{1}=\left\langle\left(s_{T_{1}}, \alpha_{1}\right),\left(s_{I_{1}}, \beta_{1}\right),\left(s_{F_{1}}, \gamma_{1}\right)\right\rangle$ and $\varphi_{2}=\left\langle\left(s_{T_{2}}, \alpha_{2}\right),\left(s_{I_{2}}, \beta_{2}\right),\left(s_{F_{2}}, \gamma_{2}\right)\right\rangle$ be two $2 T L N N$ s, then

(1) if $S\left(\varphi_{1}\right)<S\left(\varphi_{2}\right)$, then $\varphi_{1}<\varphi_{2}$;

(2) if $S\left(\varphi_{1}\right)>S\left(\varphi_{2}\right)$, then $\varphi_{1}>\varphi_{2}$;

(3) if $S\left(\varphi_{1}\right)=S\left(\varphi_{2}\right), H\left(\varphi_{1}\right)<H\left(\varphi_{2}\right)$, then $\varphi_{1}<\varphi_{2}$;

(4) if $S\left(\varphi_{1}\right)=S\left(\varphi_{2}\right), H\left(\varphi_{1}\right)>H\left(\varphi_{2}\right)$, then $\varphi_{1}>\varphi_{2}$;

(5) if $S\left(\varphi_{1}\right)=S\left(\varphi_{2}\right), H\left(\varphi_{1}\right)=H\left(\varphi_{2}\right)$, then $\varphi_{1}=\varphi_{2}$.

Definition 5. Let $\varphi_{1}=\left\langle\left(s_{T_{1}}, \alpha_{1}\right),\left(s_{I_{1}}, \beta_{1}\right),\left(s_{F_{1}}, \gamma_{1}\right)\right\rangle$ and $\varphi_{2}=\left\langle\left(s_{T_{2}}, \alpha_{2}\right),\left(s_{I_{2}}, \beta_{2}\right),\left(s_{F_{2}}, \gamma_{2}\right)\right\rangle$ be two 2 TLNNs, $\zeta>0$, then

(1) $\varphi_{1} \oplus \varphi_{2}=\left\{\begin{array}{l}\Delta\left(t\left(\frac{\Delta^{-1}\left(s_{T_{1}}, \alpha_{1}\right)}{t}+\frac{\Delta^{-1}\left(s_{T_{2}}, \alpha_{2}\right)}{t}-\frac{\Delta^{-1}\left(s_{T_{1}}, \alpha_{1}\right)}{t} \cdot \frac{\Delta^{-1}\left(s_{T_{2}}, \alpha_{2}\right)}{t}\right)\right), \\ \Delta\left(t\left(\frac{\Delta^{-1}\left(s_{I_{1}}, \beta_{1}\right)}{t} \cdot \frac{\Delta^{-1}\left(s_{I_{2}}, \beta_{2}\right)}{t}\right)\right), \Delta\left(t\left(\frac{\Delta^{-1}\left(s_{F_{1}}, \gamma_{1}\right)}{t} \cdot \frac{\Delta^{-1}\left(s_{F_{2}}, \gamma_{2}\right)}{t}\right)\right)\end{array}\right\}$; 
(2) $\varphi_{1} \otimes \varphi_{2}=\left\{\begin{array}{l}\Delta\left(t\left(\frac{\Delta^{-1}\left(s_{T_{1}}, \alpha_{1}\right)}{t} \cdot \frac{\Delta^{-1}\left(s_{T_{2}}, \alpha_{2}\right)}{t}\right)\right), \\ \Delta\left(t\left(\frac{\Delta^{-1}\left(s_{T_{1}}, \beta_{1}\right)}{t}+\frac{\Delta^{-1}\left(s_{T_{2}}, \beta_{2}\right)}{t}-\frac{\Delta^{-1}\left(s_{T_{1}}, \beta_{1}\right)}{t} \cdot \frac{\Delta^{-1}\left(s_{T_{2}}, \beta_{2}\right)}{t}\right)\right), \\ \Delta\left(t\left(\frac{\Delta^{-1}\left(s_{F_{1}}, \gamma_{1}\right)}{t}+\frac{\Delta^{-1}\left(s_{F_{2}}, \gamma_{2}\right)}{t}-\frac{\Delta^{-1}\left(s_{F_{1}}, \gamma_{1}\right)}{t} \cdot \frac{\Delta^{-1}\left(s_{F_{2}}, \gamma_{2}\right)}{t}\right)\right)\end{array}\right\} ;$

(3) $\zeta \varphi_{1}=\left\{\begin{array}{l}\Delta\left(t\left(1-\left(1-\frac{\Delta^{-1}\left(s_{T_{1}}, \alpha_{1}\right)}{t}\right)^{\zeta}\right)\right), \Delta\left(t\left(\frac{\Delta^{-1}\left(s_{I_{1}}, \beta_{1}\right)}{t}\right)^{\zeta}\right), \\ \Delta\left(t\left(\frac{\Delta^{-1}\left(s_{F_{1}}, \gamma_{1}\right)}{t}\right)^{\zeta}\right)\end{array}\right\}, \zeta>0 ;$

(4) $\quad\left(\varphi_{1}\right)^{\zeta}=\left\{\begin{array}{l}\Delta\left(t\left(\frac{\Delta^{-1}\left(s_{T_{1}}, \alpha_{1}\right)}{t}\right)^{\zeta}\right), \Delta\left(t\left(1-\left(1-\frac{\Delta^{-1}\left(s_{I_{1}}, \beta_{1}\right)}{t}\right)^{\zeta}\right)\right), \\ \Delta\left(t\left(1-\left(1-\frac{\Delta^{-1}\left(s_{F_{1}}, \gamma_{1}\right)}{t}\right)^{\zeta}\right)\right)\end{array}\right\}, \zeta>0$.

\subsection{HM Operator}

Definition 6 [32]. The Hamy mean (HM) operator is defined as follows:

$$
\operatorname{HM}^{(x)}\left(\varphi_{1}, \varphi_{2}, \cdots, \varphi_{n}\right)=\frac{\sum_{1 \leq i_{1}<\ldots<i_{x} \leq n}\left(\prod_{j=1}^{x} \varphi_{i_{j}}\right)^{\frac{1}{x}}}{C_{n}^{x}},
$$

where $x$ is a parameter and $x=1,2, \ldots, n, i_{1}, i_{2}, \ldots, i_{x}$ are $x$ integer values taken from the set $\{1,2, \ldots, n\}$ of $k$ integer values, $C_{n}^{x}$ denotes the binomial coefficient and $C_{n}^{x}=\frac{n !}{x !(n-x) !}$.

\section{Some 2TLNHM Operators}

\subsection{TLNHM Operator}

In this section, we will combine HM and 2TLNNs and propose the 2-tuple linguistic neutrosophic Hamy mean (2TLNHM) operator.

Definition 7. Let $\varphi_{j}=\left\langle\left(s_{T_{j}}, \alpha_{j}\right),\left(s_{I_{j}}, \beta_{j}\right),\left(s_{F_{j}}, \gamma_{j}\right)\right\rangle(j=1,2, \ldots, n)$ be a set of 2TLNNs. The 2TLNHM operator is

$$
2 \operatorname{TLNHM}^{(x)}\left(\varphi_{1}, \varphi_{2}, \cdots, \varphi_{n}\right)=\frac{\underset{1 \leq i_{1}<\ldots<i_{x} \leq n}{\oplus}\left(\stackrel{x}{j=1}_{j=1}^{x} \varphi_{i_{j}}\right)^{\frac{1}{x}}}{C_{n}^{x}} .
$$

Theorem 1. Let $\varphi_{j}=\left\langle\left(s_{T_{j}}, \alpha_{j}\right),\left(s_{I_{j}}, \beta_{j}\right),\left(s_{F_{j}}, \gamma_{j}\right)\right\rangle(j=1,2, \ldots, n)$ be a set of 2TLNNs. The aggregated value from the 2 TLNHM operators is also a 2 TLNN where 


$$
\begin{aligned}
& 2 \operatorname{TLNHM}^{(x)}\left(\varphi_{1}, \varphi_{2}, \cdots, \varphi_{n}\right)=\frac{\underset{1 \leq i_{1}<\ldots<i_{x} \leq n}{\oplus}\left(\substack{x \\
j=1}_{C_{n}^{x}}^{\otimes} \varphi_{i_{j}}\right)^{\frac{1}{x}}}{C_{n}^{x}} \\
& \int\left(t\left(1-\left(\prod_{1 \leq i_{1}<\ldots<i_{x} \leq n}\left(1-\left(\prod_{j=1}^{x}\left(\frac{\Delta^{-1}\left(s_{T_{j}, \alpha_{j}}\right)}{t}\right)\right)^{\frac{1}{x}}\right)\right)^{\frac{1}{C_{n}^{x}}}\right)\right), \\
& =\left\{\Delta\left(t\left(\prod_{1 \leq i_{1}<\ldots<i_{x} \leq n}\left(1-\left(\prod_{j=1}^{x}\left(1-\frac{\Delta^{-1}\left(s_{I_{j}}, \beta_{j}\right)}{t}\right)\right)^{\frac{1}{x}}\right)\right)^{\frac{1}{\mathcal{C}_{n}^{x}}}\right),\right. \\
& \Delta\left(t\left(\prod_{1 \leq i_{1}<\ldots<i_{x} \leq n}\left(1-\left(\prod_{j=1}^{x}\left(1-\frac{\Delta^{-1}\left(s_{F_{j}}, \gamma_{j}\right)}{t}\right)\right)^{\frac{1}{x}}\right)\right)^{\frac{1}{\mathcal{C}_{n}^{x}}}\right)
\end{aligned}
$$

Proof:

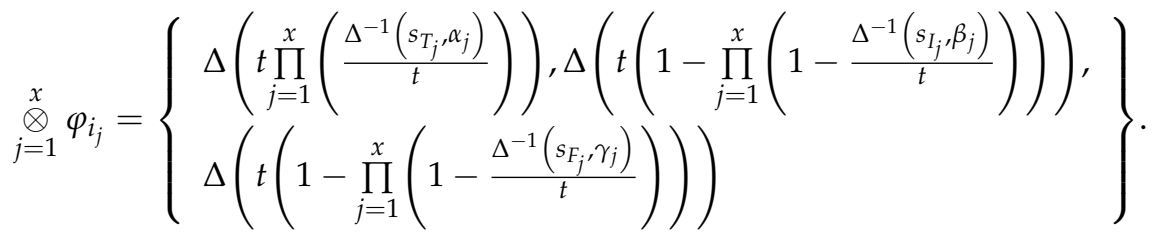

Thus,

$$
\left(\stackrel{\bigotimes}{j=1}_{j=1}^{x} \varphi_{i_{j}}\right)^{\frac{1}{x}}=\left\{\begin{array}{l}
\Delta\left(t\left(\prod_{j=1}^{x}\left(\frac{\Delta^{-1}\left(s_{T_{j},}, \alpha_{j}\right)}{t}\right)\right)^{\frac{1}{x}}\right), \Delta\left(t\left(1-\left(\prod_{j=1}^{x}\left(1-\frac{\Delta^{-1}\left(s_{I_{j},}, \beta_{j}\right)}{t}\right)\right)^{\frac{1}{x}}\right)\right), \\
\Delta\left(t\left(1-\left(\prod_{j=1}^{x}\left(1-\frac{\Delta^{-1}\left(s_{F_{j},}, \gamma_{j}\right)}{t}\right)\right)^{\frac{1}{x}}\right)\right)
\end{array}\right\}
$$

Thereafter,

$$
\underset{1 \leq i_{1}<\ldots<i_{x} \leq n}{\oplus}\left(\stackrel{\otimes}{j=1}_{j=1}^{\otimes} \varphi_{i_{j}}\right)^{\frac{1}{x}}=\left\{\begin{array}{l}
\Delta\left(t\left(1-\prod_{1 \leq i_{1}<\ldots<i_{x} \leq n}\left(1-\left(\prod_{j=1}^{x}\left(\frac{\Delta^{-1}\left(s_{T_{j}, \alpha_{j}}\right)}{t}\right)\right)^{\frac{1}{x}}\right)\right)\right), \\
\left.\Delta\left(t \prod_{1 \leq i_{1}<\ldots<i_{x} \leq n}\left(1-\left(\prod_{j=1}^{x}\left(1-\frac{\Delta^{-1}\left(s_{I_{j}, \beta_{j}}\right)}{t}\right)\right)^{\frac{1}{x}}\right)\right)\right), \\
\Delta\left(t\left(\prod_{1 \leq i_{1}<\ldots<i_{x} \leq n}\left(1-\left(\prod_{j=1}^{x}\left(1-\frac{\Delta^{-1}\left(s_{F_{j}}, \gamma_{j}\right)}{t}\right)\right)^{\frac{1}{x}}\right)\right)\right)
\end{array}\right\}
$$

Therefore, 


$$
\begin{aligned}
& 2 \operatorname{TLNHM}^{(x)}\left(\varphi_{1}, \varphi_{2}, \cdots, \varphi_{n}\right)=\frac{\underset{1 \leq i_{1}<\ldots<i_{x} \leq n}{\oplus}\left(\begin{array}{c}
x \\
\otimes \\
j=1
\end{array} \varphi_{i_{j}}\right)^{\frac{1}{x}}}{C_{n}^{x}} \\
& =\left\{\begin{array}{l}
\Delta\left(t\left(1-\left(\prod_{1 \leq i_{1}<\ldots<i_{x} \leq n}\left(1-\left(\prod_{j=1}^{x}\left(\frac{\Delta^{-1}\left(s_{T_{j}}, \alpha_{j}\right)}{t}\right)\right)^{\frac{1}{x}}\right)\right)^{\frac{1}{C_{n}^{x}}}\right)\right), \\
\left.\Delta\left(t \prod_{1 \leq i_{1}<\ldots<i_{x} \leq n}\left(1-\left(\prod_{j=1}^{x}\left(1-\frac{\Delta^{-1}\left(s_{I_{j}}, \beta_{j}\right)}{t}\right)\right)^{\frac{1}{x}}\right)\right)^{\frac{1}{C_{n}^{x}}}\right), \\
\left.\Delta\left(t \prod_{1 \leq i_{1}<\ldots<i_{x} \leq n}\left(1-\left(\prod_{j=1}^{x}\left(1-\frac{\Delta^{-1}\left(s_{F_{j},}, \gamma_{j}\right)}{t}\right)\right)^{\frac{1}{x}}\right)\right)^{\frac{1}{C_{n}^{x}}}\right)
\end{array}\right\}
\end{aligned}
$$

Hence, (7) is kept.

Then we need to prove that (7) is a 2TLNN. We need to prove two conditions, as follows:

(1) $0 \leq \Delta^{-1}\left(s_{T}, \alpha\right) \leq t, 0 \leq \Delta^{-1}\left(s_{I}, \beta\right) \leq t, 0 \leq \Delta^{-1}\left(s_{F}, \gamma\right) \leq t$.

(2) $0 \leq \Delta^{-1}\left(s_{T}, \alpha\right)+\Delta^{-1}\left(s_{I}, \beta\right)+\Delta^{-1}\left(s_{F}, \gamma\right) \leq 3 t$.

Let

$$
\begin{aligned}
& \frac{\Delta^{-1}\left(s_{T}, \alpha\right)}{t}=1-\left(\prod_{1 \leq i_{1}<\ldots<i_{x} \leq n}\left(1-\left(\prod_{j=1}^{x}\left(\frac{\Delta^{-1}\left(s_{T_{j}}, \alpha_{j}\right)}{t}\right)\right)^{\frac{1}{x}}\right)\right)^{\frac{1}{C_{n}^{x}}} \\
& \frac{\Delta^{-1}\left(s_{I}, \beta\right)}{t}=\left(\prod_{1 \leq i_{1}<\ldots<i_{x} \leq n}\left(1-\left(\prod_{j=1}^{x}\left(1-\frac{\Delta^{-1}\left(s_{I_{j}}, \beta_{j}\right)}{t}\right)\right)^{\frac{1}{x}}\right)\right)^{\frac{1}{C_{n}^{x}}} . \\
& \frac{\Delta^{-1}\left(s_{F}, \gamma\right)}{t}=\left(\prod_{1 \leq i_{1}<\ldots<i_{x} \leq n}\left(1-\left(\prod_{j=1}^{x}\left(1-\frac{\Delta^{-1}\left(s_{F_{j}}, \gamma_{j}\right)}{t}\right)\right)^{\frac{1}{x}}\right)\right)^{\frac{1}{C_{n}^{x}}}
\end{aligned}
$$

Proof: (1) Since $0 \leq \frac{\Delta^{-1}\left(s_{T_{j}}, \alpha_{j}\right)}{t} \leq 1$, we get

$$
0 \leq \prod_{j=1}^{x}\left(\frac{\Delta^{-1}\left(s_{T_{j},} \alpha_{j}\right)}{t}\right) \leq 1 \text { and } 0 \leq 1-\left(\prod_{j=1}^{x}\left(\frac{\Delta^{-1}\left(s_{T_{j},}, \alpha_{j}\right)}{t}\right)\right)^{\frac{1}{x}} \leq 1
$$

Then,

$$
\begin{gathered}
0 \leq \prod_{1 \leq i_{1}<\ldots<i_{x} \leq n}\left(1-\left(\prod_{j=1}^{x}\left(\frac{\Delta^{-1}\left(s_{T_{j}}, \alpha_{j}\right)}{t}\right)\right)^{\frac{1}{x}} \leq 1,\right. \\
0 \leq 1-\left(\prod_{1 \leq i_{1}<\ldots<i_{x} \leq n}\left(1-\left(\prod_{j=1}^{x}\left(\frac{\Delta^{-1}\left(s_{T_{j}}, \alpha_{j}\right)}{t}\right)\right)^{\frac{1}{x}}\right)\right)^{\frac{1}{C_{n}^{x}}} \leq 1 .
\end{gathered}
$$

That means $0 \leq \Delta^{-1}\left(s_{T}, \alpha\right) \leq t$. Similarly, we can get $0 \leq \Delta^{-1}\left(s_{I}, \beta\right) \leq t, 0 \leq \Delta^{-1}\left(s_{F}, \gamma\right) \leq t$ so (1) is maintained. (2) Since $0 \leq \Delta^{-1}\left(s_{T}, \alpha\right) \leq t, 0 \leq \Delta^{-1}\left(s_{I}, \beta\right) \leq t, 0 \leq \Delta^{-1}\left(s_{F}, \gamma\right) \leq t$., $0 \leq \Delta^{-1}\left(s_{T}, \alpha\right)+\Delta^{-1}\left(s_{I}, \beta\right)+\Delta^{-1}\left(s_{F}, \gamma\right) \leq 3 t$. 
Example 1. Let $\left\langle\left(s_{5}, 0\right),\left(s_{2}, 0\right),\left(s_{1}, 0\right)\right\rangle,\left\langle\left(s_{4}, 0\right),\left(s_{3}, 0\right),\left(s_{4}, 0\right)\right\rangle,\left\langle\left(s_{2}, 0\right),\left(s_{5}, 0\right),\left(s_{1}, 0\right)\right\rangle$ and $\left\langle\left(s_{5}, 0\right),\left(s_{1}, 0\right),\left(s_{3}, 0\right)\right\rangle$ be four 2 TLNNs, and suppose $x=2$, then according to (4), we have

$$
\begin{aligned}
& 2 \operatorname{TLNHM}^{(2)}\left(\varphi_{1}, \varphi_{2}, \cdots, \varphi_{n}\right)=\frac{\underset{1 \leq i_{1}<\ldots<i_{x} \leq n}{\oplus}\left(\underset{C_{n}^{x}}{\otimes} \varphi_{i=1}^{x} \varphi_{i_{j}}\right)^{\frac{1}{x}}}{C^{x}} \\
& =\left\{\begin{array}{l}
\Delta\left(\begin{array}{l}
\left.6 \times\left(\begin{array}{c}
\left(1-\left(\frac{5}{6} \times \frac{4}{6}\right)^{\frac{1}{2}}\right) \times\left(1-\left(\frac{5}{6} \times \frac{2}{6}\right)^{\frac{1}{2}}\right) \times\left(1-\left(\frac{5}{6} \times \frac{5}{6}\right)^{\frac{1}{2}}\right) \\
\times\left(1-\left(\frac{4}{6} \times \frac{2}{6}\right)^{\frac{1}{2}}\right) \times\left(1-\left(\frac{4}{6} \times \frac{5}{6}\right)^{\frac{1}{2}}\right) \times\left(1-\left(\frac{2}{6} \times \frac{5}{6}\right)^{\frac{1}{2}}\right)
\end{array}\right)\right), \\
\Delta\left(\begin{array}{l}
\left(1-\left(\left(1-\frac{2}{6}\right) \times\left(1-\frac{3}{6}\right)\right)^{\frac{1}{2}}\right) \times\left(1-\left(\left(1-\frac{2}{6}\right) \times\left(1-\frac{5}{6}\right)\right)^{\frac{1}{2}}\right) \times\left(1-\left(\left(1-\frac{2}{6}\right) \times\left(1-\frac{1}{6}\right)\right)^{\frac{1}{2}}\right) \\
\times\left(1-\left(\left(1-\frac{3}{6}\right) \times\left(1-\frac{5}{6}\right)\right)^{\frac{1}{2}}\right) \times\left(1-\left(\left(1-\frac{3}{6}\right) \times\left(1-\frac{1}{6}\right)\right)^{\frac{1}{2}}\right) \times\left(1-\left(\left(1-\frac{5}{6}\right) \times\left(1-\frac{1}{6}\right)\right)^{\frac{1}{2}}\right)
\end{array}\right), \\
\Delta\left(\begin{array}{l}
\left(1-\left(\left(1-\frac{1}{6}\right) \times\left(1-\frac{4}{6}\right)\right)^{\frac{1}{2}}\right) \times\left(1-\left(\left(1-\frac{1}{6}\right) \times\left(1-\frac{1}{6}\right)\right)^{\frac{1}{2}}\right) \times\left(1-\left(\left(1-\frac{1}{6}\right) \times\left(1-\frac{3}{6}\right)\right)^{\frac{1}{2}}\right) \\
6 \times\left(1-\left(\left(1-\frac{4}{6}\right) \times\left(1-\frac{1}{6}\right)\right)^{\frac{1}{2}}\right) \times\left(1-\left(\left(1-\frac{4}{6}\right) \times\left(1-\frac{3}{6}\right)\right)^{\frac{1}{2}}\right) \times\left(1-\left(\left(1-\frac{1}{6}\right) \times\left(1-\frac{3}{6}\right)\right)^{\frac{1}{2}}\right)
\end{array}\right)
\end{array}\right. \\
\times\left(1 \frac{1}{C_{4}^{2}}\right)
\end{array}\right\} \\
& =\left\langle\left(s_{4}, 0.0235\right),\left(s_{3},-0.1556\right),\left(s_{2}, 0.2489\right)\right\rangle
\end{aligned}
$$

Now, we will give some properties of a 2TLNHM operator.

Property 1. (Idempotency) If $\varphi_{j}=\left\langle\left(s_{T_{j}}, \alpha_{j}\right),\left(s_{I_{j}}, \beta_{j}\right),\left(s_{F_{j}}, \gamma_{j}\right)\right\rangle(j=1,2, \ldots, n)$ are equal, then

$$
2 \operatorname{TLNHM}^{(x)}\left(\varphi_{1}, \varphi_{2}, \cdots, \varphi_{n}\right)=\varphi
$$

Proof: Since $\varphi_{j}=\varphi=\left\langle\left(s_{T}, \alpha\right),\left(s_{I}, \beta\right),\left(s_{F}, \gamma\right)\right\rangle$, then

$$
\begin{aligned}
& 2 \operatorname{TLNHM}^{(x)}\left(\varphi_{1}, \varphi_{2}, \cdots, \varphi_{n}\right)=\frac{\underset{1 \leq i_{1}<\ldots<i_{x} \leq n}{\oplus}\left(\left(_{j=1}^{x} \varphi_{j} \varphi_{i_{j}}\right)^{\frac{1}{x}}\right.}{C_{n}^{x}} \\
& =\left\{\begin{array}{l}
\Delta\left(t\left(1-\left(\prod_{1 \leq i_{1}<\ldots<i_{x} \leq n}\left(1-\left(\prod_{j=1}^{x}\left(\frac{\Delta^{-1}\left(s_{T}, \alpha\right)}{t}\right)\right)^{\frac{1}{x}}\right)\right)^{\frac{1}{C_{n}^{x}}}\right)\right), \Delta\left(t\left(\prod_{1 \leq i_{1}<\ldots<i_{x} \leq n}\left(1-\left(\prod_{j=1}^{x}\left(1-\frac{\Delta^{-1}\left(s_{I}, \beta\right)}{t}\right)\right)^{\frac{1}{x}}\right)\right)^{\frac{1}{C_{n}^{x}}}\right), \\
\Delta\left(t\left(\prod_{1 \leq i_{1}<\ldots<i_{x} \leq n}\left(1-\left(\prod_{j=1}^{x}\left(1-\frac{\Delta^{-1}\left(s_{F}, \gamma\right)}{t}\right)\right)^{\frac{1}{x}}\right)\right)^{\frac{1}{C_{n}^{x}}}\right)
\end{array}\right\} \\
& =\left\{\begin{array}{l}
\Delta\left(t\left(1-\left(\left(1-\left(\left(\frac{\Delta^{-1}\left(s_{T}, \alpha\right)}{t}\right)^{x}\right)^{\frac{1}{x}}\right)^{\frac{1}{C_{n}^{x}}}\right)^{\frac{1}{C_{n}^{x}}}\right)\right), \Delta\left(t\left(\left(1-\left(\left(1-\frac{\Delta^{-1}\left(s_{I}, \beta\right)}{t}\right)^{x}\right)^{\frac{1}{x}}\right)^{\frac{1}{C_{n}^{x}}}\right)^{\frac{1}{C_{n}^{x}}}\right), \\
\Delta\left(t\left(\left(1-\left(\left(1-\frac{\Delta^{-1}\left(s_{F}, \gamma\right)}{t}\right)^{x}\right)^{\frac{1}{x}}\right)^{\frac{1}{C_{n}^{x}}}\right)^{\frac{1}{C_{n}^{x}}}\right)
\end{array}\right\} \\
& =\left\langle\left(s_{T}, \alpha\right),\left(s_{I}, \beta\right),\left(s_{F}, \gamma\right)\right\rangle=\varphi
\end{aligned}
$$

Property 2. (Monotonicity) Let $\varphi_{a_{j}}=\left\langle\left(s_{T_{a_{j}}}, \alpha_{a_{j}}\right),\left(s_{I_{a_{j}}}, \beta_{a_{j}}\right),\left(s_{F_{a_{j}}}, \gamma_{a_{j}}\right)\right\rangle(j=1,2, \ldots, n)$ and $\varphi_{b_{j}}=$ $\left\langle\left(s_{T_{b_{j}}}, \alpha_{b_{j}}\right),\left(s_{I_{b_{j}}}, \beta_{b_{j}}\right),\left(s_{F_{b_{j}}}, \gamma_{b_{j}}\right)\right\rangle(j=1,2, \ldots, n)$ be two sets of 2 TLNNs. If $\Delta^{-1}\left(s_{T_{a_{j}}}, \alpha_{a_{j}}\right) \leq$ $\Delta^{-1}\left(s_{T_{b_{j}}}, \alpha_{b_{j}}\right), \Delta^{-1}\left(s_{I_{a_{j}}}, \beta_{a_{j}}\right) \geq \Delta^{-1}\left(s_{I_{b_{j}}}, \beta_{b_{j}}\right)$ and $\Delta^{-1}\left(s_{F_{a_{j}}}, \gamma_{a_{j}}\right) \geq \Delta^{-1}\left(s_{F_{b_{j}}}, \gamma_{b_{j}}\right)$ hold for all $j$, then

$$
2 \operatorname{TLNHM}^{(x)}\left(\varphi_{a_{1}}, \varphi_{a_{2}}, \cdots, \varphi_{a_{n}}\right) \leq 2 \operatorname{TLNHM}^{(x)}\left(\varphi_{b_{1}}, \varphi_{b_{2}}, \cdots, \varphi_{b_{n}}\right)
$$


Proof: Let $\varphi_{a_{j}}=\left\langle\left(s_{T_{a_{j}}}, \alpha_{a_{j}}\right),\left(s_{I_{a_{j}}}, \beta_{a_{j}}\right),\left(s_{F_{a_{j}}}, \gamma_{a_{j}}\right)\right\rangle$ and $\varphi_{b_{j}}=\left\langle\left(s_{T_{b_{j}}}, \alpha_{b_{j}}\right),\left(s_{I_{b_{j}}}, \beta_{b_{j}}\right),\left(s_{F_{b_{j}}}, \gamma_{b_{j}}\right)\right\rangle$, given that $\Delta^{-1}\left(s_{T_{a_{j}}}, \alpha_{a_{j}}\right) \leq \Delta^{-1}\left(s_{T_{b_{j}}}, \alpha_{b_{j}}\right)$, we can obtain

$$
\begin{gathered}
\prod_{j=1}^{x}\left(\frac{\Delta^{-1}\left(s_{T_{a_{j}},} \alpha_{a_{j}}\right)}{t}\right) \leq \prod_{j=1}^{x}\left(\frac{\Delta^{-1}\left(s_{T_{b_{j}}}, \alpha_{b_{j}}\right)}{t}\right), \\
1-\left(\prod_{j=1}^{x}\left(\frac{\Delta^{-1}\left(s_{T_{a_{j}}}, \alpha_{a_{j}}\right)}{t}\right)\right)^{\frac{1}{x}} \geq 1-\left(\prod_{j=1}^{x}\left(\frac{\Delta^{-1}\left(s_{T_{b_{j}}}, \alpha_{b_{j}}\right)}{t}\right)\right)^{\frac{1}{x}} .
\end{gathered}
$$

Thereafter,

$$
\left(\prod_{1 \leq i_{1}<\ldots<i_{x} \leq n}\left(1-\left(\prod_{j=1}^{x}\left(\frac{\Delta^{-1}\left(s_{T_{j_{j}}}, \alpha_{a_{j}}\right)}{t}\right)\right)^{\frac{1}{x}}\right)\right)^{\frac{1}{C_{n}^{n}}} \geq\left(\prod_{1 \leq i_{1}<\ldots<i_{x} \leq n}\left(1-\left(\prod_{j=1}^{x}\left(\frac{\Delta^{-1}\left(s_{T_{b_{j}}, \alpha_{b_{j}}}\right)}{t}\right)\right)^{\frac{1}{x}}\right)\right)^{\frac{1}{C_{n}^{x}}}
$$

Furthermore,

$$
1-\left(\prod_{1 \leq i_{1}<\ldots<i_{x} \leq n}\left(1-\left(\prod_{j=1}^{x}\left(\frac{\Delta^{-1}\left(s_{T_{a_{j}}, \alpha_{a_{j}}}\right)}{t}\right)\right)^{\frac{1}{x}}\right)\right)^{\frac{1}{C_{n}^{x}}} \leq 1-\left(\prod_{1 \leq i_{1}<\ldots<i_{x} \leq n}\left(1-\left(\prod_{j=1}^{x}\left(\frac{\Delta^{-1}\left(s_{T_{b_{j}}, \alpha_{b_{j}}}\right)}{t}\right)\right)^{\frac{1}{x}}\right)\right)^{\frac{1}{C_{n}^{n}}} .
$$

That means $\Delta^{-1}\left(s_{T_{a}}, \alpha_{a}\right) \leq \Delta^{-1}\left(s_{T_{b}}, \alpha_{b}\right)$. Similarly, we can obtain $\Delta^{-1}\left(s_{I_{a}}, \beta_{a}\right) \geq \Delta^{-1}\left(s_{I_{b}}, \beta_{b}\right)$ and $\Delta^{-1}\left(s_{F_{a}}, \gamma_{a}\right) \geq \Delta^{-1}\left(s_{F_{b}}, \gamma_{b}\right)$.

$$
\begin{aligned}
& \text { If } \Delta^{-1}\left(s_{T_{a}}, \alpha_{a}\right)<\Delta^{-1}\left(s_{T_{b}}, \alpha_{b}\right), \Delta^{-1}\left(s_{I_{a}}, \beta_{a}\right) \geq \Delta^{-1}\left(s_{I_{b}}, \beta_{b}\right) \text { and } \Delta^{-1}\left(s_{F_{a}}, \gamma_{a}\right) \geq \Delta^{-1}\left(s_{F_{b}}, \gamma_{b}\right), \\
& 2 \operatorname{TLNHM}^{(x)}\left(\varphi_{a}, \varphi_{a}, \cdots, \varphi_{a}\right)<2 \operatorname{TLNHM}^{(x)}\left(\varphi_{b}, \varphi_{b}, \cdots, \varphi_{b}\right) \\
& \text { If } \Delta^{-1}\left(s_{T_{a}}, \alpha_{a}\right)=\Delta^{-1}\left(s_{T_{b}}, \alpha_{b}\right), \Delta^{-1}\left(s_{I_{a}}, \beta_{a}\right)>\Delta^{-1}\left(s_{I_{b}}, \beta_{b}\right) \text { and } \Delta^{-1}\left(s_{F_{a}}, \gamma_{a}\right)>\Delta^{-1}\left(s_{F_{b}}, \gamma_{b}\right), \\
& 2 \operatorname{TLNHM}^{(x)}\left(\varphi_{a}, \varphi_{a}, \cdots, \varphi_{a}\right)<2 \operatorname{TLNHM}^{(x)}\left(\varphi_{b}, \varphi_{b}, \cdots, \varphi_{b}\right) \\
& \text { If } \Delta^{-1}\left(s_{T_{a}}, \alpha_{a}\right)=\Delta^{-1}\left(s_{T_{b}}, \alpha_{b}\right), \Delta^{-1}\left(s_{I_{a}}, \beta_{a}\right)=\Delta^{-1}\left(s_{I_{b}}, \beta_{b}\right) \text { and } \Delta^{-1}\left(s_{F_{a}}, \gamma_{a}\right)=\Delta^{-1}\left(s_{F_{b}}, \gamma_{b}\right), \\
& 2 \operatorname{TLNHM}^{(x)}\left(\varphi_{a}, \varphi_{a}, \cdots, \varphi_{a}\right)=2 \operatorname{TLNHM}^{(x)}\left(\varphi_{b}, \varphi_{b}, \cdots, \varphi_{b}\right) \\
& \text { So, Property } 2 \text { is right. } \square
\end{aligned}
$$

Property 3. (Boundedness) Let $\varphi_{j}=\left\langle\left(s_{T_{j}}, \alpha_{j}\right),\left(s_{I_{j}}, \beta_{j}\right),\left(s_{F_{j}}, \gamma_{j}\right)\right\rangle(j=1,2, \ldots, n)$ be $a$ set of 2 TLNNs. If $\varphi_{i}^{+}=\left(\max _{i}\left(s_{T_{j}}, \alpha_{j}\right), \min _{i}\left(s_{I_{j}}, \beta_{j}\right), \min _{i}\left(s_{F_{j}}, \gamma_{j}\right)\right)$ and $\varphi_{i}^{+}=$ $\left(\max _{i}\left(s_{T_{j}}, \alpha_{j}\right), \min _{i}\left(s_{I_{j}}, \beta_{j}\right), \min _{i}\left(s_{F_{j}}, \gamma_{j}\right)\right)$, then

$$
\varphi^{-} \leq 2 \operatorname{TLNHM}^{(x)}\left(\varphi_{1}, \varphi_{2}, \cdots, \varphi_{n}\right) \leq \varphi^{+} .
$$

From Property 1,

$$
\begin{aligned}
& 2 \operatorname{TLNHM}^{(x)}\left(\varphi_{1}^{-}, \varphi_{2}^{-}, \cdots, \varphi_{n}^{-}\right)=\varphi^{-} \\
& 2 \operatorname{TLNHM}^{(x)}\left(\varphi_{1}^{+}, \varphi_{2}^{+}, \cdots, \varphi_{n}^{+}\right)=\varphi^{+}
\end{aligned} .
$$

From Property 2,

$$
\varphi^{-} \leq 2 \operatorname{TLNHM}^{(x)}\left(\varphi_{1}, \varphi_{2}, \cdots, \varphi_{n}\right) \leq \varphi^{+} .
$$

\subsection{The 2TLNWHM Operator}

In an actual MADM, it is important to consider attribute weights. This section proposes a 2-tuple linguistic neutrosophic weighted Hamy mean (2TLNWHM) operator as follows. 
Definition 8. Let $\varphi_{j}=\left\langle\left(s_{T_{j}}, \alpha_{j}\right),\left(s_{I_{j}}, \beta_{j}\right),\left(s_{F_{j}}, \gamma_{j}\right)\right\rangle(j=1,2, \ldots, n)$ be a set of $2 T L N N$ s with a weight vector, $w_{i}=\left(w_{1}, w_{2}, \ldots, w_{n}\right)^{T}$, thereby satisfying $w_{i} \in[0,1]$ and $\sum_{i=1}^{n} w_{i}=1$. Then, we can define the 2TLNWHM operator as follows:

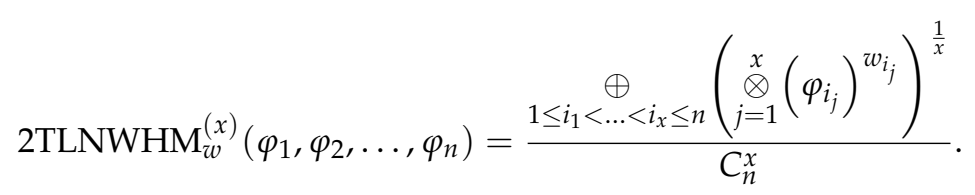

Theorem 2. Let $\varphi_{j}=\left\langle\left(s_{T_{j}}, \alpha_{j}\right),\left(s_{I_{j}}, \beta_{j}\right),\left(s_{F_{j}}, \gamma_{j}\right)\right\rangle(j=1,2, \ldots, n)$ be a set of 2 TLNNs. The aggregated value determined using a 2 TLNWHM operator is also a 2 TLNN, where

$$
\begin{aligned}
& 2 \operatorname{TLNWHM}_{w}^{(x)}\left(\varphi_{1}, \varphi_{2}, \ldots, \varphi_{n}\right)=\frac{\underset{1 \leq i_{1}<\ldots<i_{x} \leq n}{\oplus}\left(\underset{\substack{x \\
j=1}}{\otimes}\left(\varphi_{i_{j}}\right)^{w_{i_{j}}}\right)^{\frac{1}{x}}}{C_{n}^{x}} \\
& =\left\{\begin{array}{l}
\Delta\left(t\left(1-\left(\prod_{1 \leq i_{1}<\ldots<i_{x} \leq n}\left(1-\left(\prod_{j=1}^{x}\left(\frac{\Delta^{-1}\left(s_{T_{j},}, \alpha_{j}\right)}{t}\right)^{w_{i_{j}}}\right)^{\frac{1}{x}}\right)\right)^{\frac{1}{C_{n}^{x}}}\right)\right), \\
\left.\Delta\left(t \prod_{1 \leq i_{1}<\ldots<i_{x} \leq n}\left(1-\left(\prod_{j=1}^{x}\left(1-\frac{\Delta^{-1}\left(s_{I_{j}}, \beta_{j}\right)}{t}\right)^{w_{i_{j}}}\right)^{\frac{1}{x}}\right)\right)^{\frac{1}{C_{n}^{x}}}\right), \\
\left.\Delta\left(t \prod_{1 \leq i_{1}<\ldots<i_{x} \leq n}\left(1-\left(\prod_{j=1}^{x}\left(1-\frac{\Delta^{-1}\left(s_{F_{j},}, \gamma_{j}\right)}{t}\right)^{w_{i_{j}}}\right)^{\frac{1}{x}}\right)\right)^{\frac{1}{C_{n}^{x}}}\right)
\end{array}\right\} .
\end{aligned}
$$

Proof: From Definition 5, we can obtain,

$$
\left(\varphi_{i_{j}}\right)^{w_{i_{j}}}=\left\{\begin{array}{l}
\Delta\left(t\left(\frac{\Delta^{-1}\left(s_{T_{j}}, \alpha_{j}\right)}{t}\right)^{w_{i_{j}}}\right), \Delta\left(t\left(1-\left(1-\frac{\Delta^{-1}\left(s_{I_{j}}, \beta_{j}\right)}{t}\right)^{w_{i_{j}}}\right)\right) \\
\Delta\left(t\left(1-\left(1-\frac{\Delta^{-1}\left(s_{F_{j}}, \gamma_{j}\right)}{t}\right)^{w_{i_{j}}}\right)\right)
\end{array}\right\} .
$$

Thus,

$$
\stackrel{\bigotimes}{j=1}_{j=1}^{x}\left(\varphi_{i_{j}}\right)^{w_{i_{j}}}=\left\{\begin{array}{l}
\Delta\left(t\left(\prod_{j=1}^{x}\left(\frac{\Delta^{-1}\left(s_{\left.T_{j^{\prime}}, \alpha_{j}\right)}\right)}{t}\right)^{w_{i_{j}}}\right)\right), \Delta\left(t\left(1-\prod_{j=1}^{x}\left(1-\frac{\Delta^{-1}\left(s_{I_{j},}, \beta_{j}\right)}{t}\right)^{w_{i_{j}}}\right)\right), \\
\Delta\left(t\left(1-\prod_{j=1}^{x}\left(1-\frac{\Delta^{-1}\left(s_{F_{j}}, \gamma_{j}\right)}{t}\right)^{w_{i_{j}}}\right)\right)
\end{array}\right\}
$$

Therefore,

$$
\left(\stackrel{\bigotimes}{j=1}_{j=1}^{\otimes}\left(\varphi_{i_{j}}\right)^{w_{i_{j}}}\right)^{\frac{1}{x}}=\left\{\begin{array}{l}
\Delta\left(t\left(\prod_{j=1}^{x}\left(\frac{\Delta^{-1}\left(s_{T_{j}, \alpha_{j}}\right)}{t}\right)^{w_{i_{j}}}\right)^{\frac{1}{x}}\right), \Delta\left(t\left(1-\left(\prod_{j=1}^{x}\left(1-\frac{\Delta^{-1}\left(s_{s_{j}}, \beta_{j}\right)}{t}\right)^{w_{i_{j}}}\right)^{\frac{1}{x}}\right)\right), \\
\Delta\left(t\left(1-\left(\prod_{j=1}^{x}\left(1-\frac{\Delta^{-1}\left(s_{F_{j}}, \gamma_{j}\right)}{t}\right)^{w_{i_{j}}}\right)^{\frac{1}{x}}\right)\right)
\end{array}\right\} .
$$


Thereafter,

$$
\underset{1 \leq i_{1}<\ldots<i_{x} \leq n}{\oplus}\left(\stackrel{x}{j=1}_{j=1}^{\otimes}\left(\varphi_{i_{j}}\right)^{w_{i_{j}}}\right)^{\frac{1}{x}}=\left\{\begin{array}{c}
\Delta\left(t\left(1-\prod_{1 \leq i_{1}<\ldots<i_{x} \leq n}\left(1-\left(\prod_{j=1}^{x}\left(\frac{\Delta^{-1}\left(s_{T_{j}, \alpha_{j}}\right)}{t}\right)^{w_{i_{j}}}\right)^{\frac{1}{x}}\right)\right)\right), \\
\left.\Delta\left(\prod_{1 \leq i_{1}<\ldots<i_{x} \leq n}\left(1-\left(\prod_{j=1}^{x}\left(1-\frac{\Delta^{-1}\left(s_{I_{j}, \beta_{j}}\right)}{t}\right)^{w_{i_{j}}}\right)^{\frac{1}{x}}\right)\right)\right), \\
\Delta\left(t\left(\prod_{1 \leq i_{1}<\ldots<i_{x} \leq n}\left(1-\left(\prod_{j=1}^{x}\left(1-\frac{\Delta^{-1}\left(s_{F_{j}}, \gamma_{j}\right)}{t}\right)^{w_{w_{j}}}\right)^{\frac{1}{x}}\right)\right)\right)
\end{array}\right\}
$$

Furthermore,

$$
\begin{aligned}
& 2 \operatorname{TLNWHM}_{w}^{(x)}\left(\varphi_{1}, \varphi_{2}, \ldots, \varphi_{n}\right)=\frac{\underset{1 \leq i_{1}<\ldots<i_{x} \leq n}{\oplus}\left(\underset{\substack{x \\
j=1}}{\otimes}\left(\varphi_{i_{j}}\right)^{w_{i j}}\right)^{\frac{1}{x}}}{C_{n}^{x}} . \\
& =\left\{\begin{array}{l}
\Delta\left(t\left(1-\left(\prod_{1 \leq i_{1}<\ldots<i_{x} \leq n}\left(1-\left(\prod_{j=1}^{x}\left(\frac{\Delta^{-1}\left(s_{T_{j}, \alpha_{j}}\right)}{t}\right)^{w_{i_{j}}}\right)^{\frac{1}{x}}\right)\right)^{\frac{1}{C_{n}^{x}}}\right)\right), \\
\left.\Delta\left(t \prod_{1 \leq i_{1}<\ldots<i_{x} \leq n}\left(1-\left(\prod_{j=1}^{x}\left(1-\frac{\Delta^{-1}\left(s_{I_{j}}, \beta_{j}\right)}{t}\right)^{w_{i_{j}}}\right)^{\frac{1}{x}}\right)\right)^{\frac{1}{C_{n}^{x}}}\right), \\
\left.\Delta\left(t \prod_{1 \leq i_{1}<\ldots<i_{x} \leq n}\left(1-\left(\prod_{j=1}^{x}\left(1-\frac{\Delta^{-1}\left(s_{F_{j}}, \gamma_{j}\right)}{t}\right)^{w_{i_{j}}}\right)^{\frac{1}{x}}\right)\right)^{\frac{1}{C_{n}^{x}}}\right)
\end{array}\right\} .
\end{aligned}
$$

Hence, (23) is kept.

Then we need to prove that (23) is a 2 TLNN. We need to prove two conditions as follows:

(1) $0 \leq \Delta^{-1}\left(s_{T}, \alpha\right) \leq t, 0 \leq \Delta^{-1}\left(s_{I}, \beta\right) \leq t, 0 \leq \Delta^{-1}\left(s_{F}, \gamma\right) \leq t$.

(2) $0 \leq \Delta^{-1}\left(s_{T}, \alpha\right)+\Delta^{-1}\left(s_{I}, \beta\right)+\Delta^{-1}\left(s_{F}, \gamma\right) \leq 3 t$.

Let

$$
\begin{aligned}
\frac{\Delta^{-1}\left(s_{T}, \alpha\right)}{t} & =1-\left(\prod_{1 \leq i_{1}<\ldots<i_{x} \leq n}\left(1-\left(\prod_{j=1}^{x}\left(\frac{\Delta^{-1}\left(s_{T_{j}}, \alpha_{j}\right)}{t}\right)^{w_{i_{j}}}\right)^{\frac{1}{x}}\right)\right)^{\frac{1}{C_{n}^{x}}} \\
\frac{\Delta^{-1}\left(s_{I}, \beta\right)}{t} & =\left(\prod_{1 \leq i_{1}<\ldots<i_{x} \leq n}\left(1-\left(\prod_{j=1}^{x}\left(1-\frac{\Delta^{-1}\left(s_{I_{j}}, \beta_{j}\right)}{t}\right)^{w_{i_{j}}}\right)^{\frac{1}{x}}\right)\right)^{\frac{1}{C_{n}^{x}}} \\
\frac{\Delta^{-1}\left(s_{F}, \gamma\right)}{t} & =\left(\prod_{1 \leq i_{1}<\ldots<i_{x} \leq n}\left(1-\left(\prod_{j=1}^{x}\left(1-\frac{\Delta^{-1}\left(s_{F_{j}}, \gamma_{j}\right)}{t}\right)^{w_{i_{j}}}\right)^{\frac{1}{x}}\right)\right)^{\frac{1}{C_{n}^{x}}} .
\end{aligned}
$$


Proof. (1) Since $0 \leq \frac{\Delta^{-1}\left(s_{T_{j}}, \alpha_{j}\right)}{t} \leq 1$, we get

$$
0 \leq \prod_{j=1}^{x}\left(\frac{\Delta^{-1}\left(s_{T_{j}}, \alpha_{j}\right)}{t}\right)^{w_{i_{j}}} \leq 1 \text { and } 0 \leq 1-\left(\prod_{j=1}^{x}\left(\frac{\Delta^{-1}\left(s_{T_{j}}, \alpha_{j}\right)}{t}\right)^{w_{i_{j}}}\right)^{\frac{1}{x}} \leq 1
$$

Then,

$$
\begin{array}{r}
0 \leq \prod_{1 \leq i_{1}<\ldots<i_{x} \leq n}\left(1-\left(\prod_{j=1}^{x}\left(\frac{\Delta^{-1}\left(s_{T_{j}}, \alpha_{j}\right)}{t}\right)^{w_{i_{j}}}\right)^{\frac{1}{x}} \leq 1\right. \\
0 \leq 1-\left(\prod_{1 \leq i_{1}<\ldots<i_{x} \leq n}\left(1-\left(\prod_{j=1}^{x}\left(\frac{\Delta^{-1}\left(s_{T_{j}}, \alpha_{j}\right)}{t}\right)^{w_{i_{j}}}\right)^{\frac{1}{x}}\right)\right)^{\frac{1}{C_{n}^{x}}} \leq 1
\end{array}
$$

That means $0 \leq \Delta^{-1}\left(s_{T}, \alpha\right) \leq t$. Similarly, we can get $0 \leq \Delta^{-1}\left(s_{I}, \beta\right) \leq t, 0 \leq \Delta^{-1}\left(s_{F}, \gamma\right) \leq t$. So, (1) is maintained; (2) Since $0 \leq \Delta^{-1}\left(s_{T}, \alpha\right) \leq t, 0 \leq \Delta^{-1}\left(s_{I}, \beta\right) \leq t, 0 \leq \Delta^{-1}\left(s_{F}, \gamma\right) \leq t$. $0 \leq \Delta^{-1}\left(s_{T}, \alpha\right)+\Delta^{-1}\left(s_{I}, \beta\right)+\Delta^{-1}\left(s_{F}, \gamma\right) \leq 3 t$.

Example 2. Let $\left\langle\left(s_{5}, 0\right),\left(s_{2}, 0\right),\left(s_{1}, 0\right)\right\rangle,\left\langle\left(s_{4}, 0\right),\left(s_{3}, 0\right),\left(s_{4}, 0\right)\right\rangle,\left\langle\left(s_{2}, 0\right),\left(s_{5}, 0\right),\left(s_{1}, 0\right)\right\rangle$ and $\left\langle\left(s_{5}, 0\right),\left(s_{1}, 0\right),\left(s_{3}, 0\right)\right\rangle$ be four 2TLNNs, $w=(0.2,0.3,0.4,0.1)$ and suppose $x=2$, then according to (23), we have

$$
\begin{aligned}
& 2 \operatorname{TLNHM}^{(2)}\left(\varphi_{1}, \varphi_{2}, \cdots, \varphi_{n}\right)=\frac{\underset{1 \leq i_{1}<\ldots \ldots i_{x} \leq n}{\oplus}\left(\sum_{j=1}^{x}\left(\varphi_{i_{j}}\right)^{w_{i_{j}}}\right)^{\frac{1}{x}}}{C_{n}^{1}}
\end{aligned}
$$

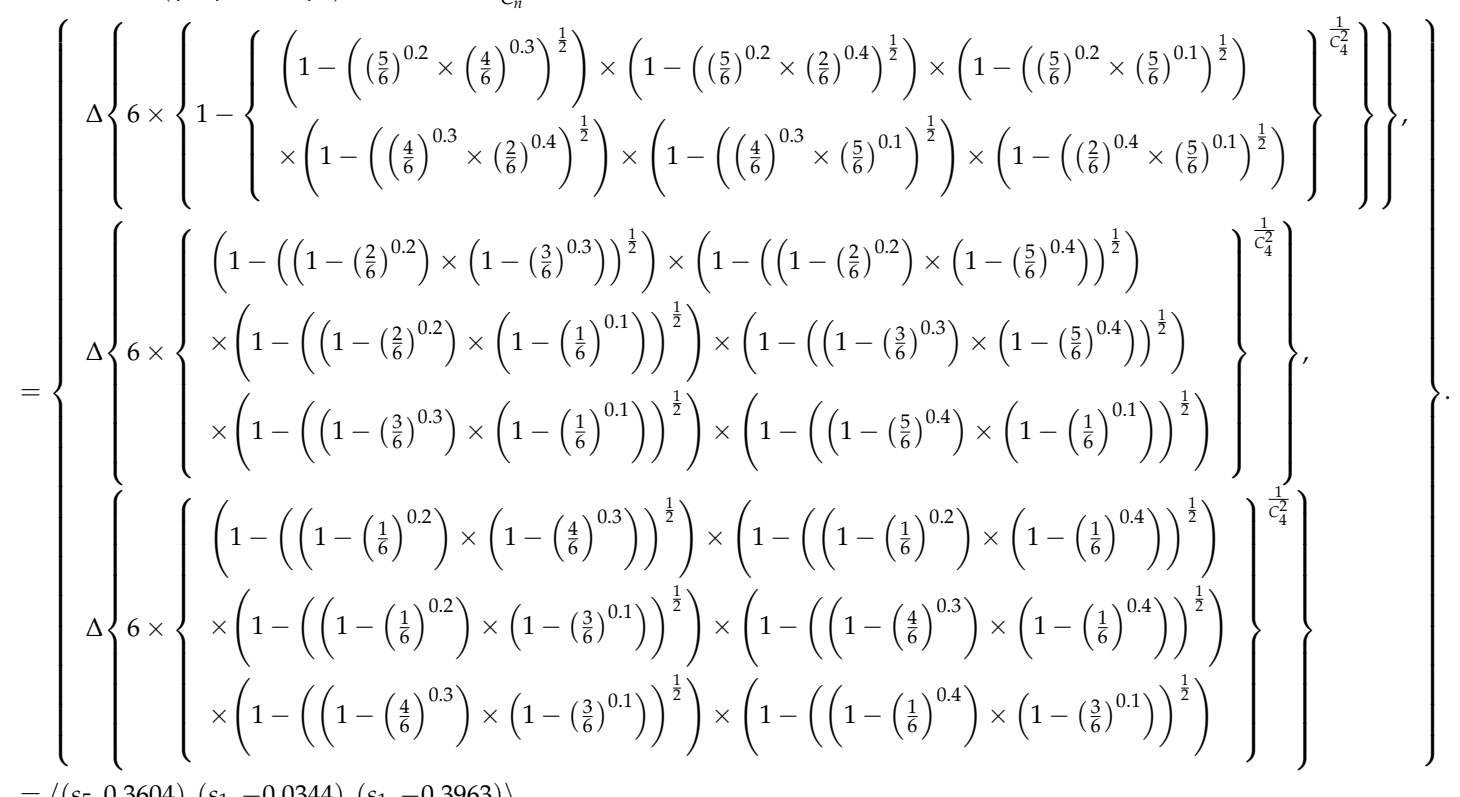

$$
\begin{aligned}
& =\left\langle\left(s_{5}, 0.3604\right),\left(s_{1},-0.0344\right),\left(s_{1},-0.3963\right)\right\rangle
\end{aligned}
$$

Now, we will discuss some properties of the 2TLNWHM operator. 
Property 4. (Monotonicity) Let $\varphi_{a_{j}}=\left\langle\left(s_{T_{a_{j}}}, \alpha_{a_{j}}\right),\left(s_{I_{a_{j}}}, \beta_{a_{j}}\right),\left(s_{F_{a_{j}}}, \gamma_{a_{j}}\right)\right\rangle(j=1,2, \ldots, n)$ and $\varphi_{b_{j}}=$ $\left\langle\left(s_{T_{b}}, \alpha_{b_{j}}\right),\left(s_{I_{b_{j}}}, \beta_{b_{j}}\right),\left(s_{F_{b_{j}}}, \gamma_{b_{j}}\right)\right\rangle(j=1,2, \ldots, n)$ be two sets of 2 TLNNs. If $\Delta^{-1}\left(s_{T_{a_{j}}}, \alpha_{a_{j}}\right) \leq$ $\Delta^{-1}\left(s_{T_{b_{j}}}, \alpha_{b_{j}}\right), \Delta^{-1}\left(s_{I_{a_{j}}}, \beta_{a_{j}}\right) \geq \Delta^{-1}\left(s_{I_{b_{j}}}, \beta_{b_{j}}\right)$ and $\Delta^{-1}\left(s_{F_{a_{j}}}, \gamma_{a_{j}}\right) \geq \Delta^{-1}\left(s_{F_{b_{j}}}, \gamma_{b_{j}}\right)$ hold for all $j$, then

$$
2 \operatorname{TLNWHM}^{(x)}\left(\varphi_{a_{1}}, \varphi_{a_{2}}, \cdots, \varphi_{a_{n}}\right) \leq 2 \operatorname{TLNWHM}^{(x)}\left(\varphi_{b_{1}}, \varphi_{b_{2}}, \cdots, \varphi_{b_{n}}\right)
$$

The proof is similar to 2TLNWHM; it is omitted here.

Property 5. (Boundedness) Let $\varphi_{j}=\left\langle\left(s_{T_{j}}, \alpha_{j}\right),\left(s_{I_{j}}, \beta_{j}\right),\left(s_{F_{j}}, \gamma_{j}\right)\right\rangle(j=1,2, \ldots, n)$ be $a$ set of 2 TLNNs. If $\varphi_{i}^{+}=\left(\max _{i}\left(s_{T_{j}}, \alpha_{j}\right), \min _{i}\left(s_{I_{j}}, \beta_{j}\right), \min _{i}\left(s_{F_{j}}, \gamma_{j}\right)\right)$ and $\varphi_{i}^{+}=$ $\left(\max _{i}\left(s_{T_{j}}, \alpha_{j}\right), \min _{i}\left(s_{I_{j}}, \beta_{j}\right), \min _{i}\left(s_{F_{j}}, \gamma_{j}\right)\right)$, then

$$
\varphi^{-} \leq 2 \operatorname{TLNWHM}^{(x)}\left(\varphi_{1}, \varphi_{2}, \cdots, \varphi_{n}\right) \leq \varphi^{+} .
$$

From theorem 2, we get

$$
\begin{aligned}
& 2 \operatorname{TLNWHM}_{w}^{(x)}\left(\left(\varphi_{1}^{-}, \varphi_{2}^{-}, \ldots, \varphi_{n}^{-}\right)\right)=\frac{\left.\underset{1 \leq i_{1}<\ldots<i_{x} \leq n}{\oplus}\left(\underset{\underset{j}{x}\left(\min \varphi_{i_{j}}\right.}{{ }^{w}}\right)^{w_{i_{j}}}\right)^{\frac{1}{x}}}{C_{n}^{x}} \\
& =\left\{\begin{array}{c}
\Delta\left(t\left(1-\left(\prod_{1 \leq i_{1}<\ldots<i_{x} \leq n}\left(1-\left(\prod_{j=1}^{x}\left(\frac{\min \Delta^{-1}\left(s_{T_{j}}, \alpha_{j}\right)}{t}\right)^{w_{i_{j}}}\right)^{\frac{1}{x}}\right)\right)^{\frac{1}{C_{n}^{x}}}\right)\right), \\
\left.\Delta\left(\prod_{1 \leq i_{1}<\ldots<i_{x} \leq n}\left(1-\left(\prod_{j=1}^{x}\left(1-\frac{\max \Delta^{-1}\left(s_{I_{j}}, \beta_{j}\right)}{t}\right)^{w_{i_{j}}}\right)^{\frac{1}{x}}\right)\right)^{\frac{1}{C_{n}^{x}}}\right), \\
\left.\Delta\left(t \prod_{1 \leq i_{1}<\ldots<i_{x} \leq n}\left(1-\left(\prod_{j=1}^{x}\left(1-\frac{\max \Delta^{-1}\left(s_{F_{j}}, \gamma_{j}\right)}{t}\right)^{w_{i_{j}}}\right)^{\frac{1}{x}}\right)\right)^{\frac{1}{C_{n}^{x}}}\right)
\end{array}\right\},
\end{aligned}
$$

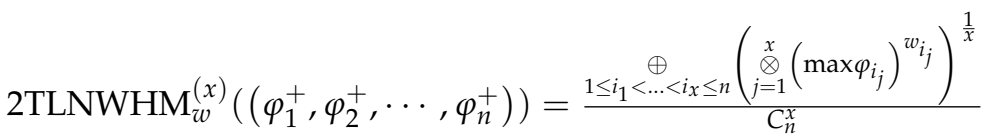

$$
\begin{aligned}
& =\left\{\begin{array}{l}
\Delta\left(t\left(1-\left(\prod_{1 \leq i_{1}<\ldots<i_{x} \leq n}\left(1-\left(\prod_{j=1}^{x}\left(\frac{\max \Delta^{-1}\left(s_{T_{j}}, \alpha_{j}\right)}{t}\right)^{w_{i_{j}}}\right)^{\frac{1}{x}}\right)\right)^{\frac{1}{C_{n}^{x}}}\right)\right), \\
\Delta\left(t\left(\prod_{1 \leq i_{1}<\ldots<i_{x} \leq n}\left(1-\left(\prod_{j=1}^{x}\left(1-\frac{\min \Delta^{-1}\left(s_{I_{j}}, \beta_{j}\right)}{t}\right)^{w_{i_{j}}}\right)^{\frac{1}{x}}\right)\right)^{\frac{1}{C_{n}^{x}}}\right), \\
\left.\Delta\left(t \prod_{1 \leq i_{1}<\ldots<i_{x} \leq n}\left(1-\left(\prod_{j=1}^{x}\left(1-\frac{\min \Delta^{-1}\left(s_{F_{j}}, \gamma_{j}\right)}{t}\right)^{w_{i_{j}}}\right)^{\frac{1}{x}}\right)\right)^{\frac{1}{C_{n}^{x}}}\right)
\end{array}\right\} .
\end{aligned}
$$


From property 4, we get

$$
\varphi^{-} \leq 2 \operatorname{TLNWHM}^{(x)}\left(\varphi_{1}, \varphi_{2}, \cdots, \varphi_{n}\right) \leq \varphi^{+}
$$

It is obvious that the 2TLNWHM operator lacks the property of idempotency.

\subsection{The 2TLNDHM Operator}

Based on the Hamy mean (HM) operator [32], we propose the dual Hamy mean (DHM) operator.

Definition 9. The DHM operator is defined as follows:

$$
\operatorname{DHM}^{(x)}\left(\varphi_{1}, \varphi_{2}, \cdots, \varphi_{n}\right)=\left(\prod_{1 \leq i_{1}<\ldots<i_{x} \leq n}\left(\frac{\sum_{j=1}^{x} \varphi_{i_{j}}}{x}\right)\right)^{\frac{1}{C_{n}^{x}}} .
$$

where $x$ is a parameter and $x=1,2, \ldots, n, i_{1}, i_{2}, \ldots, i_{x}$ are $x$ integer values taken from the set $\{1,2, \ldots, n\}$ of $k$ integer values, $C_{n}^{x}$ denotes the binomial coefficient and $C_{n}^{x}=\frac{n !}{x !(n-x) !}$.

In this section, we propose the 2-tuple linguistic neutrosophic DHM (2TLNDHM) operator.

Definition 10. Let $\varphi_{j}=\left\langle\left(s_{T_{j}}, \alpha_{j}\right),\left(s_{I_{j}}, \beta_{j}\right),\left(s_{F_{j}}, \gamma_{j}\right)\right\rangle(j=1,2, \ldots, n)$ be a set of 2 TLNNs. The 2TLNDHM operator is:

$$
\operatorname{TLLNDHM}^{(x)}\left(\varphi_{1}, \varphi_{2}, \cdots, \varphi_{n}\right)=\left(\underset{1 \leq i_{1}<\ldots<i_{x} \leq n}{\otimes}\left(\frac{\stackrel{x}{\oplus} \varphi_{i_{j}}}{x}\right)\right)^{\frac{1}{\mathcal{C}_{n}^{x}}} .
$$

Theorem 3. Let $\varphi_{j}=\left\langle\left(s_{T_{j}}, \alpha_{j}\right),\left(s_{I_{j}}, \beta_{j}\right),\left(s_{F_{j}}, \gamma_{j}\right)\right\rangle(j=1,2, \ldots, n)$ be a set of 2 TLNNs. The aggregated value determined using 2 TLNDHM operators is also a 2 TLNN where

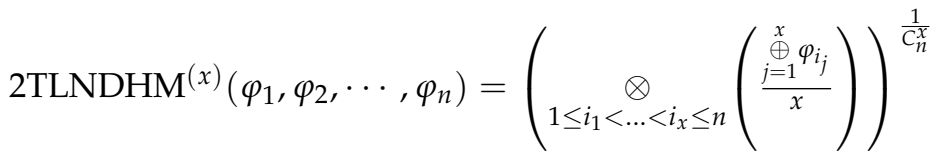

$$
\begin{aligned}
& =\left\{\begin{array}{l}
\Delta\left(t\left(\prod_{1 \leq i_{1}<\ldots<i_{x} \leq n}\left(1-\left(\prod_{j=1}^{x}\left(1-\frac{\Delta^{-1}\left(s_{T_{j},}, \alpha_{j}\right)}{t}\right)\right)^{\frac{1}{x}}\right)\right)^{\frac{1}{C_{n}^{x}}}\right), \\
\left.\Delta\left(1-\left(\prod_{1 \leq i_{1}<\ldots<i_{x} \leq n}\left(1-\left(\prod_{j=1}^{x}\left(\frac{\Delta^{-1}\left(s_{I_{j}}, \beta_{j}\right)}{t}\right)\right)^{\frac{1}{x}}\right)\right)^{\frac{1}{C_{n}^{x}}}\right)\right), \\
\Delta\left(t\left(1-\left(\prod_{1 \leq i_{1}<\ldots<i_{x} \leq n}\left(1-\left(\prod_{j=1}^{x}\left(\frac{\Delta^{-1}\left(s_{F_{j}}, \gamma_{j}\right)}{t}\right)\right)^{\frac{1}{x}}\right)\right)^{\frac{1}{C_{n}^{x}}}\right)\right)
\end{array}\right\}
\end{aligned}
$$




\section{Proof:}

$$
\bigoplus_{j=1}^{x} \varphi_{i_{j}}=\left\{\begin{array}{l}
\Delta\left(t\left(1-\prod_{j=1}^{x}\left(1-\frac{\Delta^{-1}\left(s_{T_{j}}, \alpha_{j}\right)}{t}\right)\right)\right), \Delta\left(t\left(\prod_{j=1}^{x}\left(\frac{\Delta^{-1}\left(s_{I_{j}}, \beta_{j}\right)}{t}\right)\right)\right), \\
\Delta\left(t\left(\prod_{j=1}^{x}\left(\frac{\Delta^{-1}\left(s_{F_{j}}, \gamma_{j}\right)}{t}\right)\right)\right)
\end{array}\right\}
$$

Thus,

$$
\frac{\bigoplus_{j=1}^{x} \varphi_{i_{j}}}{x}=\left\{\begin{array}{l}
\Delta\left(t\left(1-\left(\prod_{j=1}^{x}\left(1-\frac{\Delta^{-1}\left(s_{T_{j}}, \alpha_{j}\right)}{t}\right)\right)^{\frac{1}{x}}\right)\right), \Delta\left(t\left(\prod_{j=1}^{x}\left(\frac{\Delta^{-1}\left(s_{I_{j},} \beta_{j}\right)}{t}\right)\right)^{\frac{1}{x}}\right), \\
\Delta\left(t\left(\prod_{j=1}^{x}\left(\frac{\Delta^{-1}\left(s_{F_{j}}, \gamma_{j}\right)}{t}\right)\right)^{\frac{1}{x}}\right)
\end{array}\right\}
$$

Thereafter,

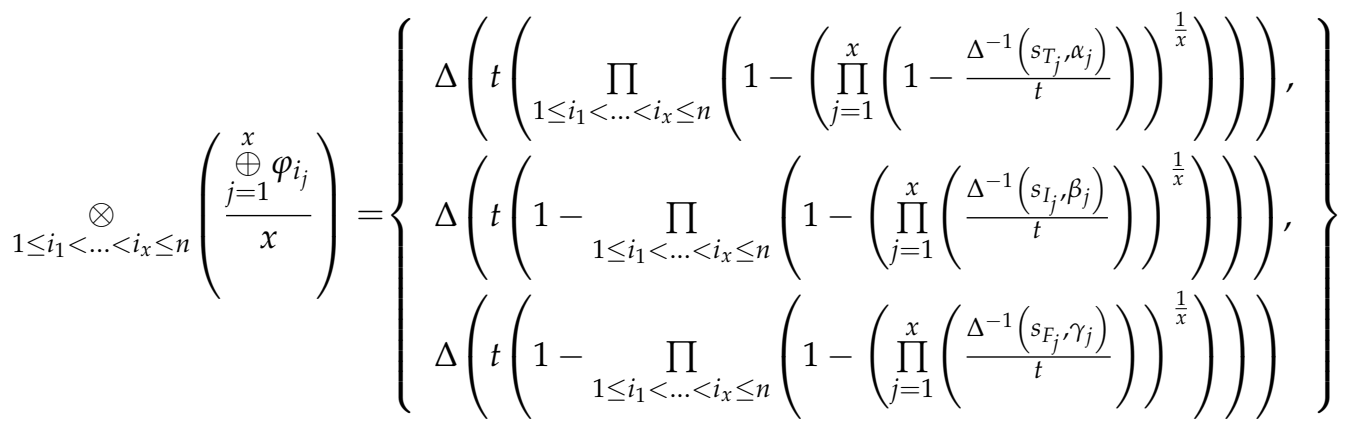

Therefore,

$$
\begin{aligned}
& 2 \operatorname{TLNDHM}^{(x)}\left(\varphi_{1}, \varphi_{2}, \cdots, \varphi_{n}\right)=\left(\underset{1 \leq i_{1}<\ldots<i_{x} \leq n}{\otimes}\left(\begin{array}{c}
\underset{j=1}{\oplus} \varphi_{i_{j}} \\
x
\end{array}\right)\right)^{\frac{1}{\mathrm{C}_{n}^{x}}} \\
& =\left\{\begin{array}{l}
\left.\Delta\left(t \prod_{1 \leq i_{1}<\ldots<i_{x} \leq n}\left(1-\left(\prod_{j=1}^{x}\left(1-\frac{\Delta^{-1}\left(s_{T_{j},}, \alpha_{j}\right)}{t}\right)\right)^{\frac{1}{x}}\right)\right)^{\frac{1}{C_{n}^{x}}}\right), \\
\Delta\left(t\left(1-\left(\prod_{1 \leq i_{1}<\ldots<i_{x} \leq n}\left(1-\left(\prod_{j=1}^{x}\left(\frac{\Delta^{-1}\left(s_{\left.I_{j}, \beta_{j}\right)}\right)}{t}\right)\right)^{\frac{1}{x}}\right)\right)^{\frac{1}{C_{n}^{x}}}\right)\right), \\
\Delta\left(t\left(1-\left(\prod_{1 \leq i_{1}<\ldots<i_{x} \leq n}\left(1-\left(\prod_{j=1}^{x}\left(\frac{\Delta^{-1}\left(s_{F_{j},}, \gamma_{j}\right)}{t}\right)\right)^{\frac{1}{x}}\right)\right)^{\frac{1}{C_{n}^{x}}}\right)\right.
\end{array} .\right.
\end{aligned}
$$

Hence, (39) is kept.

Then, we need to prove that (39) is a 2TLNN. We need to prove two conditions as follows:

(1) $\quad 0 \leq \Delta^{-1}\left(s_{T}, \alpha\right) \leq t, 0 \leq \Delta^{-1}\left(s_{I}, \beta\right) \leq t, 0 \leq \Delta^{-1}\left(s_{F}, \gamma\right) \leq t$.

(2) $0 \leq \Delta^{-1}\left(s_{T}, \alpha\right)+\Delta^{-1}\left(s_{I}, \beta\right)+\Delta^{-1}\left(s_{F}, \gamma\right) \leq 3 t$. 
Let

$$
\begin{aligned}
& \frac{\Delta^{-1}\left(s_{T}, \alpha\right)}{t}=\left(\prod_{1 \leq i_{1}<\ldots<i_{x} \leq n}\left(1-\left(\prod_{j=1}^{x}\left(1-\frac{\Delta^{-1}\left(s_{T_{j}}, \alpha_{j}\right)}{t}\right)\right)^{\frac{1}{x}}\right)\right)^{\frac{1}{C_{n}^{x}}} \\
& \frac{\Delta^{-1}\left(s_{I}, \beta\right)}{t}=1-\left(\prod_{1 \leq i_{1}<\ldots<i_{x} \leq n}\left(1-\left(\prod_{j=1}^{x}\left(\frac{\Delta^{-1}\left(s_{I_{j}}, \beta_{j}\right)}{t}\right)\right)^{\frac{1}{x}}\right)\right)^{\frac{1}{C_{n}^{x}}} \\
& \frac{\Delta^{-1}\left(s_{F}, \gamma\right)}{t}=1-\left(\prod_{1 \leq i_{1}<\ldots<i_{x} \leq n}\left(1-\left(\prod_{j=1}^{x}\left(\frac{\Delta^{-1}\left(s_{F_{j}}, \gamma_{j}\right)}{t}\right)\right)^{\frac{1}{x}}\right)\right)^{\frac{1}{C_{n}^{x}}}
\end{aligned}
$$

Proof. (1) Since $0 \leq \frac{\Delta^{-1}\left(s_{T_{j}}, \alpha_{j}\right)}{t} \leq 1$, we get

$$
0 \leq \prod_{j=1}^{x}\left(1-\frac{\Delta^{-1}\left(s_{T_{j}}, \alpha_{j}\right)}{t}\right) \leq 1 \text { and } 0 \leq 1-\left(\prod_{j=1}^{x}\left(1-\frac{\Delta^{-1}\left(s_{T_{j},}, \alpha_{j}\right)}{t}\right)\right)^{\frac{1}{x}} \leq 1 .
$$

Then,

$$
\begin{gathered}
0 \leq \prod_{1 \leq i_{1}<\ldots<i_{x} \leq n}\left(1-\left(\prod_{j=1}^{x}\left(1-\frac{\Delta^{-1}\left(s_{T_{j}}, \alpha_{j}\right)}{t}\right)\right)^{\frac{1}{x}} \leq 1\right. \\
0 \leq\left(\prod_{1 \leq i_{1}<\ldots<i_{x} \leq n}\left(1-\left(\prod_{j=1}^{x}\left(1-\frac{\Delta^{-1}\left(s_{T_{j}}, \alpha_{j}\right)}{t}\right)\right)^{\frac{1}{x}}\right)\right)^{\frac{1}{C_{n}^{x}}} \leq 1
\end{gathered}
$$

That means $0 \leq \Delta^{-1}\left(s_{T}, \alpha\right) \leq t$. Similarly, we can get $0 \leq \Delta^{-1}\left(s_{I}, \beta\right) \leq t, 0 \leq \Delta^{-1}\left(s_{F}, \gamma\right) \leq t$. So, (1) is maintained. (2) Since $0 \leq \Delta^{-1}\left(s_{T}, \alpha\right) \leq t, 0 \leq \Delta^{-1}\left(s_{I}, \beta\right) \leq t, 0 \leq \Delta^{-1}\left(s_{F}, \gamma\right) \leq t$. $0 \leq \Delta^{-1}\left(s_{T}, \alpha\right)+\Delta^{-1}\left(s_{I}, \beta\right)+\Delta^{-1}\left(s_{F}, \gamma\right) \leq 3 t$.

Example 3. Let $\left\langle\left(s_{5}, 0\right),\left(s_{2}, 0\right),\left(s_{1}, 0\right)\right\rangle,\left\langle\left(s_{4}, 0\right),\left(s_{3}, 0\right),\left(s_{4}, 0\right)\right\rangle,\left\langle\left(s_{2}, 0\right),\left(s_{5}, 0\right),\left(s_{1}, 0\right)\right\rangle \quad$ and $\left\langle\left(s_{5}, 0\right),\left(s_{1}, 0\right),\left(s_{3}, 0\right)\right\rangle$ be four $2 T L N N$ s, and suppose $x=2$, then according to (39), we have

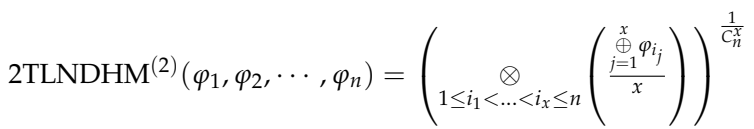

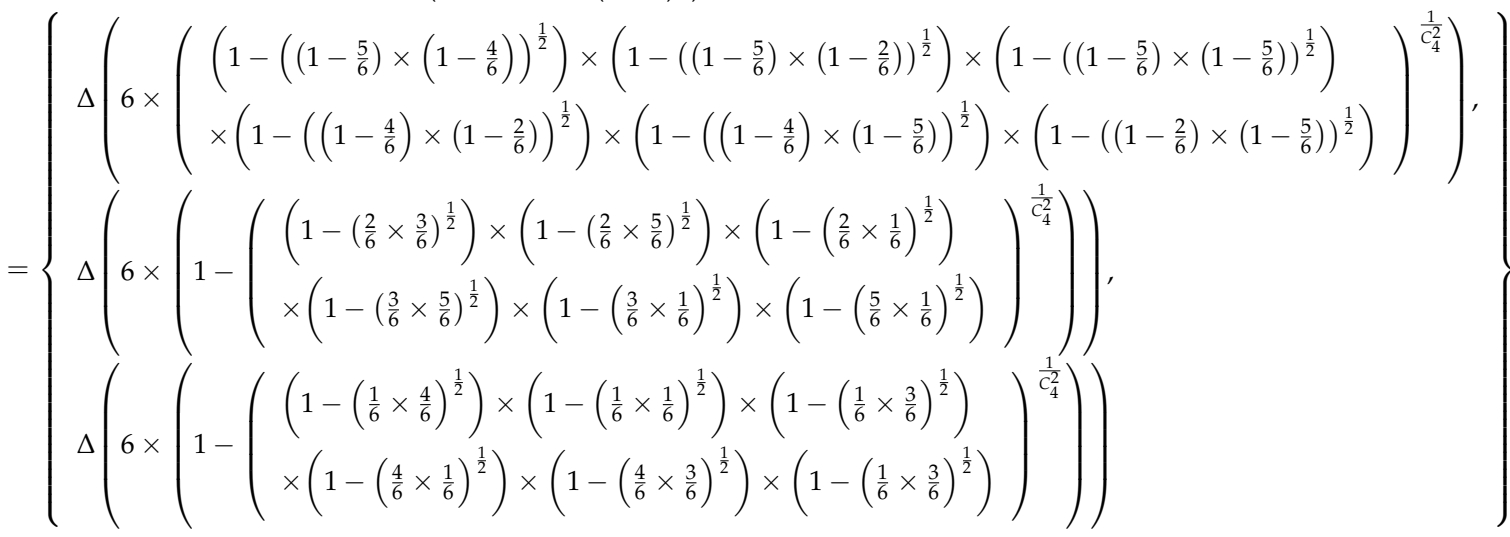

$$
\begin{aligned}
& =\left\langle\left(s_{4}, 0.1802\right),\left(s_{3},-0.4123\right),\left(s_{2}, 0.0680\right)\right\rangle
\end{aligned}
$$


Similar to the 2TLNHM operator, we can get the properties, as follows.

Property 6. (Idempotency) If $\varphi_{j}=\left\langle\left(s_{T_{j}}, \alpha_{j}\right),\left(s_{I_{j}}, \beta_{j}\right),\left(s_{F_{j}}, \gamma_{j}\right)\right\rangle(j=1,2, \ldots, n)$ are equal, then

$$
2 \operatorname{TLNDHM}^{(x)}\left(\varphi_{1}, \varphi_{2}, \cdots, \varphi_{n}\right)=\varphi \text {. }
$$

Property 7. (Monotonicity) Let $\varphi_{a_{j}}=\left\langle\left(s_{T_{a_{j}}}, \alpha_{a_{j}}\right),\left(s_{I_{a_{j}}}, \beta_{a_{j}}\right),\left(s_{F_{a_{j}}}, \gamma_{a_{j}}\right)\right\rangle(j=1,2, \ldots, n)$ and $\varphi_{b_{j}}=$ $\left\langle\left(s_{T_{b_{j}}}, \alpha_{b_{j}}\right),\left(s_{I_{b_{j}}}, \beta_{b_{j}}\right),\left(s_{F_{b_{j}}}, \gamma_{b_{j}}\right)\right\rangle(j=1,2, \ldots, n)$ be two sets of 2 TLNNs. If $\Delta^{-1}\left(s_{T_{a_{j}}}, \alpha_{a_{j}}\right) \leq$ $\Delta^{-1}\left(s_{T_{b_{j}}}, \alpha_{b_{j}}\right), \Delta^{-1}\left(s_{I_{a_{j}}}, \beta_{a_{j}}\right) \geq \Delta^{-1}\left(s_{I_{b_{j}}}, \beta_{b_{j}}\right)$ and $\Delta^{-1}\left(s_{F_{a_{j}}}, \gamma_{a_{j}}\right) \geq \Delta^{-1}\left(s_{F_{b_{j}}}, \gamma_{b_{j}}\right)$ hold for all $j$, then

$$
2 \operatorname{TLNDHM}^{(x)}\left(\varphi_{a_{1}}, \varphi_{a_{2}}, \cdots, \varphi_{a_{n}}\right) \leq 2 \operatorname{TLNDHM}^{(x)}\left(\varphi_{b_{1}}, \varphi_{b_{2}}, \cdots, \varphi_{b_{n}}\right)
$$

Property 8. (Boundedness) Let $\varphi_{j}=\left\langle\left(s_{T_{j}}, \alpha_{j}\right),\left(s_{I_{j}}, \beta_{j}\right),\left(s_{F_{j}}, \gamma_{j}\right)\right\rangle(j=1,2, \ldots, n)$ be a set of 2TLNNs. If $\varphi_{i}^{+}=\left(\max _{i}\left(s_{T_{j}}, \alpha_{j}\right), \min _{i}\left(s_{I_{j}}, \beta_{j}\right), \min _{i}\left(s_{F_{j}}, \gamma_{j}\right)\right)$ and $\varphi_{i}^{+}=$ $\left(\max _{i}\left(s_{T_{j}}, \alpha_{j}\right), \min _{i}\left(s_{I_{j}}, \beta_{j}\right), \min _{i}\left(s_{F_{j}}, \gamma_{j}\right)\right)$ then

$$
\varphi^{-} \leq 2 \operatorname{TLNDHM}^{(x)}\left(\varphi_{1}, \varphi_{2}, \cdots, \varphi_{n}\right) \leq \varphi^{+} .
$$

\subsection{The 2TLNWDHM Operator}

In an actual MADM, it is important to consider attribute weights; in this section we shall propose the 2-tuple linguistic neutrosophic weighted DHM (2TLNWDHM) operator.

Definition 11. Let $\varphi_{j}=\left\langle\left(s_{T_{j}}, \alpha_{j}\right),\left(s_{I_{j}}, \beta_{j}\right),\left(s_{F_{j}}, \gamma_{j}\right)\right\rangle(j=1,2, \ldots, n)$ be a set of 2 TLNNs with the weight vector, $w_{i}=\left(w_{1}, w_{2}, \ldots, w_{n}\right)^{T}$, thereby satisfying $w_{i} \in[0,1]$ and $\sum_{i=1}^{n} w_{i}=1$. If

$$
2 \operatorname{TLNWDHM}^{(x)}\left(\varphi_{1}, \varphi_{2}, \cdots, \varphi_{n}\right)=\left(\underset{1 \leq i_{1}<\ldots<i_{x} \leq n}{\otimes}\left(\frac{\underset{j=1}{\oplus} w_{i_{j}} \varphi_{i_{j}}}{x}\right)\right)^{\frac{1}{C_{n}^{x}}} .
$$


Theorem 4. Let $\varphi_{j}=\left\langle\left(s_{T_{j}}, \alpha_{j}\right),\left(s_{I_{j}}, \beta_{j}\right),\left(s_{F_{j}}, \gamma_{j}\right)\right\rangle(j=1,2, \ldots, n)$ be a set of 2 TLNNs. The aggregated value by using 2 TLNWDHM operators is also a $2 T L N N$ where

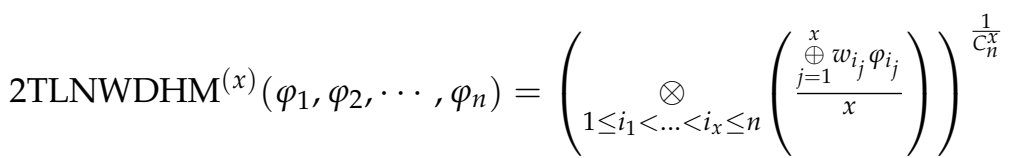

$$
\begin{aligned}
& =\left\{\begin{array}{l}
\Delta\left(t\left(\prod_{1 \leq i_{1}<\ldots<i_{x} \leq n}\left(1-\left(\prod_{j=1}^{x}\left(1-\frac{\Delta^{-1}\left(s_{T_{j},} \alpha_{j}\right)}{t}\right)^{w_{i_{j}}}\right)^{\frac{1}{x}}\right)\right)^{\frac{1}{C_{n}^{x}}}\right), \\
\left.\Delta\left(1-\left(\prod_{1 \leq i_{1}<\ldots<i_{x} \leq n}\left(1-\left(\prod_{j=1}^{x}\left(\frac{\Delta^{-1}\left(s_{I_{j},} \beta_{j}\right)}{t}\right)^{w_{i_{j}}}\right)^{\frac{1}{x}}\right)\right)^{\frac{1}{C_{n}^{x}}}\right)\right), \\
\Delta\left(t\left(1-\left(\prod_{1 \leq i_{1}<\ldots<i_{x} \leq n}\left(1-\left(\prod_{j=1}^{x}\left(\frac{\Delta^{-1}\left(s_{F_{j}}, \gamma_{j}\right)}{t}\right)^{w_{i_{j}}}\right)^{\frac{1}{x}}\right)\right)^{\frac{1}{C_{n}^{x}}}\right)\right)
\end{array}\right\}
\end{aligned}
$$

Proof. From Definition 5, we can obtain that

$$
w_{i_{j}} \varphi_{i_{j}}=\left\{\begin{array}{l}
\Delta\left(t\left(1-\left(1-\frac{\Delta^{-1}\left(s_{T_{j}}, \alpha_{j}\right)}{t}\right)^{w_{i_{j}}}\right)\right) \\
\Delta\left(t\left(\frac{\Delta^{-1}\left(s_{I_{j}}, \beta_{j}\right)}{t}\right)^{w_{i_{j}}}\right), \Delta\left(t\left(\frac{\Delta^{-1}\left(s_{F_{j}}, \gamma_{j}\right)}{t}\right)^{w_{i_{j}}}\right)
\end{array}\right\}
$$

Then,

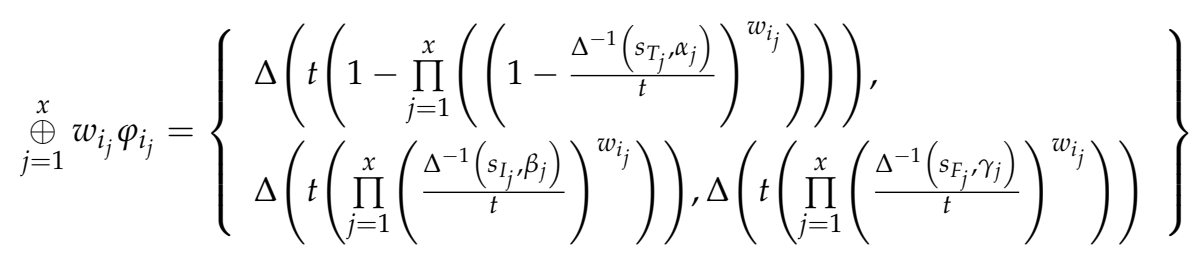

Thus,

$$
\frac{\bigoplus_{j=1}^{x} w_{i_{j}} \varphi_{i_{j}}}{x}=\left\{\begin{array}{l}
\Delta\left(t\left(1-\left(\prod_{j=1}^{x}\left(1-\frac{\Delta^{-1}\left(s_{T_{j}}, \alpha_{j}\right)}{t}\right)^{w_{i_{j}}}\right)^{\frac{1}{x}}\right)\right), \\
\Delta\left(t\left(\prod_{j=1}^{x}\left(\frac{\Delta^{-1}\left(s_{I_{j},}, \beta_{j}\right)}{t}\right)^{w_{i_{j}}}\right)^{\frac{1}{x}}\right), \Delta\left(t\left(\prod_{j=1}^{x}\left(\frac{\Delta^{-1}\left(s_{F_{j}}, \gamma_{j}\right)}{t}\right)^{w_{i_{j}}}\right)^{\frac{1}{x}}\right)
\end{array}\right\}
$$


Therefore,

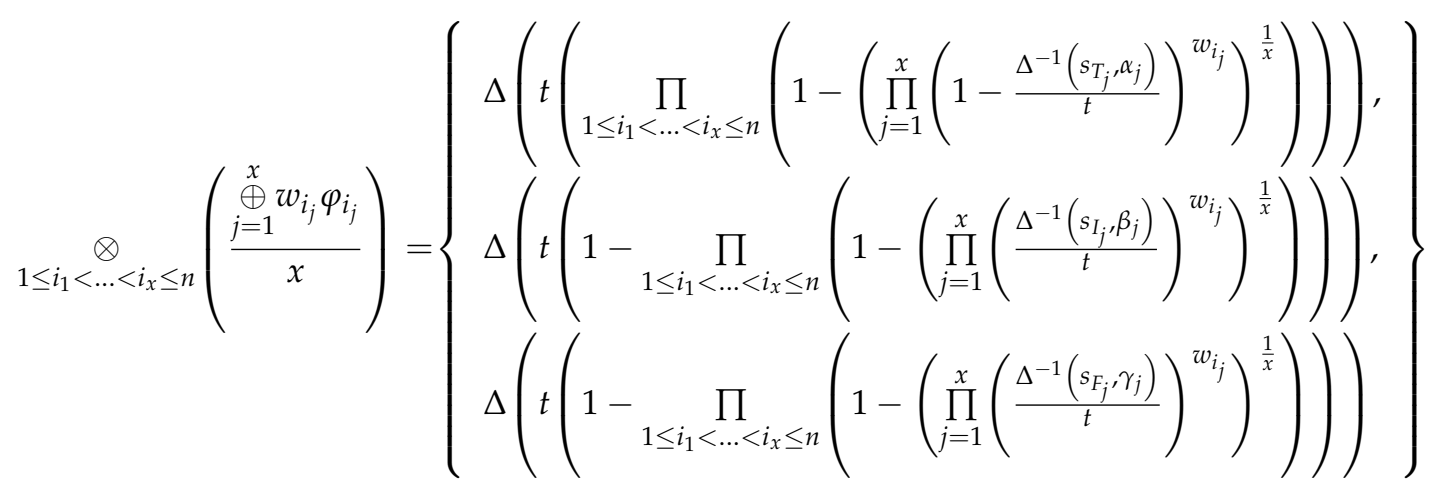

Therefore,

$$
\begin{aligned}
& 2 \operatorname{TLNWDHM}^{(x)}\left(\varphi_{1}, \varphi_{2}, \cdots, \varphi_{n}\right)=\left(\underset{1 \leq i_{1}<\ldots<i_{x} \leq n}{\otimes}\left(\frac{\stackrel{x}{\oplus} \underset{j=1}{\otimes} w_{i j} \varphi_{i_{j}}}{x}\right)\right)^{\frac{1}{C_{n}^{x}}} \\
& =\left\{\begin{array}{l}
\Delta\left(t\left(\prod_{1 \leq i_{1}<\ldots<i_{x} \leq n}\left(1-\left(\prod_{j=1}^{x}\left(1-\frac{\Delta^{-1}\left(s_{T_{j},} \alpha_{j}\right)}{t}\right)^{w_{i_{j}}}\right)^{\frac{1}{x}}\right)\right)^{\frac{1}{C_{n}^{x}}}\right), \\
\left.\Delta\left(t 1-\left(\prod_{1 \leq i_{1}<\ldots<i_{x} \leq n}\left(1-\left(\prod_{j=1}^{x}\left(\frac{\Delta^{-1}\left(s_{I_{j},} \beta_{j}\right)}{t}\right)^{w_{i_{j}}}\right)^{\frac{1}{x}}\right)\right)^{\frac{1}{C_{n}^{x}}}\right)\right), \\
\Delta\left(t\left(1-\left(\prod_{1 \leq i_{1}<\ldots<i_{x} \leq n}^{\prod_{j=1}}\left(1-\left(\prod_{j=1}^{x}\left(\frac{\Delta^{-1}\left(s_{F_{j}, \gamma_{j}}\right)}{t}\right)^{w_{i_{j}}}\right)^{\frac{1}{x}}\right)\right)^{\frac{1}{C_{n}^{x}}}\right)\right)
\end{array}\right\}
\end{aligned}
$$

Hence, (51) is kept.

Then, we need to prove that (51) is a 2 TLNN. We need to prove two conditions as follows:

(1) $0 \leq \Delta^{-1}\left(s_{T}, \alpha\right) \leq t, 0 \leq \Delta^{-1}\left(s_{I}, \beta\right) \leq t, 0 \leq \Delta^{-1}\left(s_{F}, \gamma\right) \leq t$.

(2) $0 \leq \Delta^{-1}\left(s_{T}, \alpha\right)+\Delta^{-1}\left(s_{I}, \beta\right)+\Delta^{-1}\left(s_{F}, \gamma\right) \leq 3 t$.

Let

$$
\begin{aligned}
& \frac{\Delta^{-1}\left(s_{T}, \alpha\right)}{t}=\left(\prod_{1 \leq i_{1}<\ldots<i_{x} \leq n}\left(1-\left(\prod_{j=1}^{x}\left(1-\frac{\Delta^{-1}\left(s_{T_{j}}, \alpha_{j}\right)}{t}\right)^{w_{i_{j}}}\right)^{\frac{1}{x}}\right)\right)^{\frac{1}{C_{n}^{x}}} \\
& \frac{\Delta^{-1}\left(s_{I}, \beta\right)}{t}=1-\left(\prod_{1 \leq i_{1}<\ldots<i_{x} \leq n}\left(1-\left(\prod_{j=1}^{x}\left(\frac{\Delta^{-1}\left(s_{I_{j}}, \beta_{j}\right)}{t}\right)^{w_{i_{j}}}\right)^{\frac{1}{x}}\right)\right)^{\frac{1}{C_{n}^{x}}} \\
& \frac{\Delta^{-1}\left(s_{F}, \gamma\right)}{t}=1-\left(\prod_{1 \leq i_{1}<\ldots<i_{x} \leq n}\left(1-\left(\prod_{j=1}^{x}\left(\frac{\Delta^{-1}\left(s_{F_{j}}, \gamma_{j}\right)}{t}\right)^{w_{i_{j}}}\right)^{\frac{1}{x}}\right)\right)^{\frac{1}{C_{n}^{x}}}
\end{aligned}
$$


Proof. (1) Since $0 \leq \frac{\Delta^{-1}\left(s_{T_{j}}, \alpha_{j}\right)}{t} \leq 1$, we get

$$
0 \leq \prod_{j=1}^{x}\left(1-\frac{\Delta^{-1}\left(s_{T_{j}}, \alpha_{j}\right)}{t}\right) \leq 1 \text { and } 0 \leq 1-\left(\prod_{j=1}^{x}\left(1-\frac{\Delta^{-1}\left(s_{T_{j}}, \alpha_{j}\right)}{t}\right)^{w_{i_{j}}}\right)^{\frac{1}{x}} \leq 1
$$

Then,

$$
\begin{gathered}
0 \leq \prod_{1 \leq i_{1}<\ldots<i_{x} \leq n}\left(1-\left(\prod_{j=1}^{x}\left(1-\frac{\Delta^{-1}\left(s_{T_{j}}, \alpha_{j}\right)}{t}\right)^{w_{i_{j}}}\right)^{\frac{1}{x}} \leq 1\right. \\
0 \leq\left(\prod_{1 \leq i_{1}<\ldots<i_{x} \leq n}\left(1-\left(\prod_{j=1}^{x}\left(1-\frac{\Delta^{-1}\left(s_{T_{j}}, \alpha_{j}\right)}{t}\right)^{w_{i_{j}}}\right)^{\frac{1}{x}}\right)\right)^{\frac{1}{C_{n}^{x}}} \leq 1 .
\end{gathered}
$$

That means $0 \leq \Delta^{-1}\left(s_{T}, \alpha\right) \leq t$. Similarly, we can get $0 \leq \Delta^{-1}\left(s_{I}, \beta\right) \leq t, 0 \leq \Delta^{-1}\left(s_{F}, \gamma\right) \leq t$ so (1) is maintained. (2) Since $0 \leq \Delta^{-1}\left(s_{T}, \alpha\right) \leq t, 0 \leq \Delta^{-1}\left(s_{I}, \beta\right) \leq t, 0 \leq \Delta^{-1}\left(s_{F}, \gamma\right) \leq t$. $0 \leq \Delta^{-1}\left(s_{T}, \alpha\right)+\Delta^{-1}\left(s_{I}, \beta\right)+\Delta^{-1}\left(s_{F}, \gamma\right) \leq 3 t$.

Example 4. Let $\left\langle\left(s_{5}, 0\right),\left(s_{2}, 0\right),\left(s_{1}, 0\right)\right\rangle,\left\langle\left(s_{4}, 0\right),\left(s_{3}, 0\right),\left(s_{4}, 0\right)\right\rangle,\left\langle\left(s_{2}, 0\right),\left(s_{5}, 0\right),\left(s_{1}, 0\right)\right\rangle$ and $\left\langle\left(s_{5}, 0\right),\left(s_{1}, 0\right),\left(s_{3}, 0\right)\right\rangle$ be four 2TLNNs, $w=(0.2,0.3,0.4,0.1)$ and suppose $x=2$, then according to (51), we have

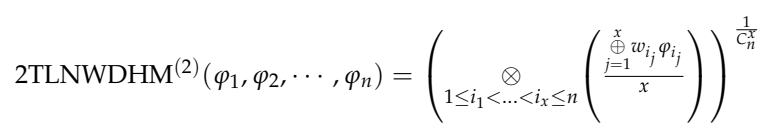

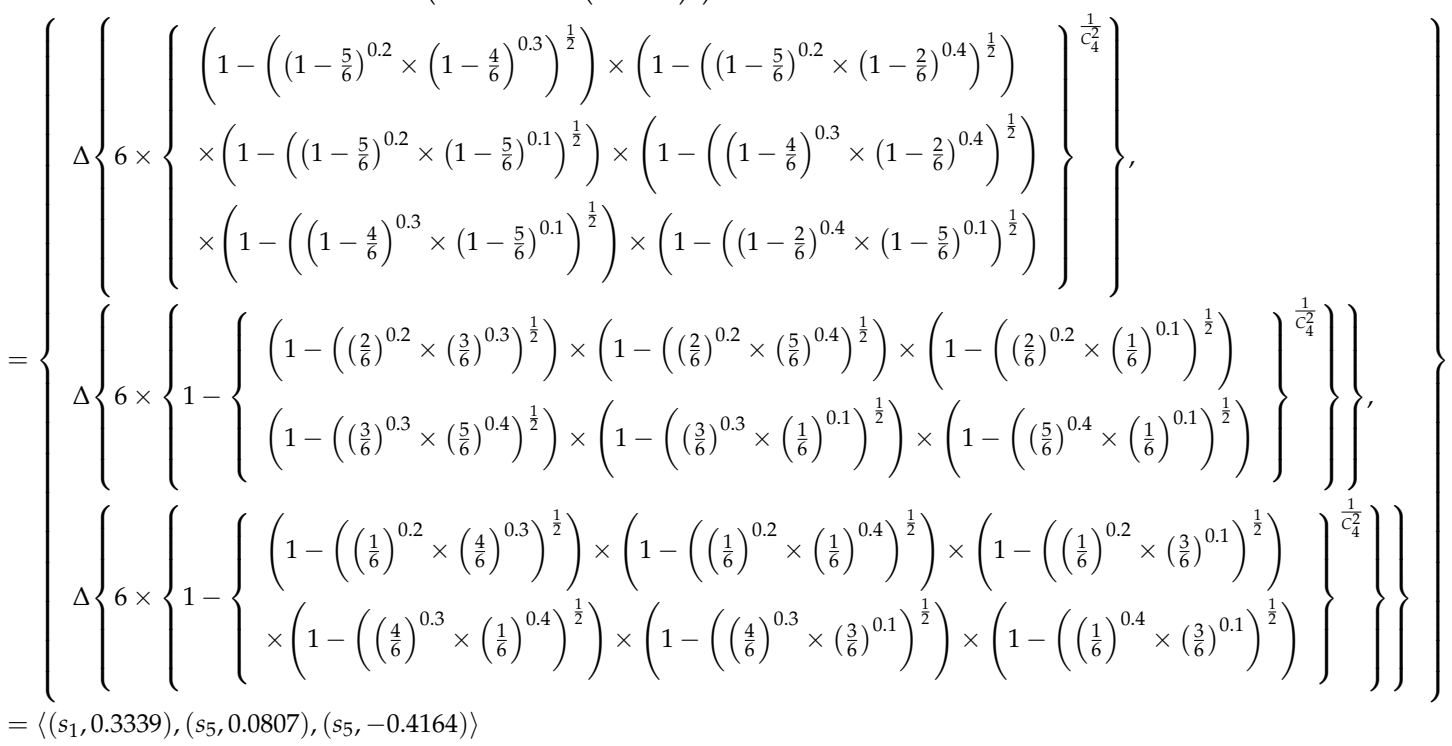

Now, we will discuss some properties of the 2TLNWDHM operator. 
Property 9. (Monotonicity) Let $\varphi_{a_{j}}=\left\langle\left(s_{T_{a_{j}}}, \alpha_{a_{j}}\right),\left(s_{I_{a_{j}}}, \beta_{a_{j}}\right),\left(s_{F_{a_{j}}}, \gamma_{a_{j}}\right)\right\rangle(j=1,2, \ldots, n)$ and $\varphi_{b_{j}}=$ $\left\langle\left(s_{T_{b}}, \alpha_{b_{j}}\right),\left(s_{I_{b_{j}}}, \beta_{b_{j}}\right),\left(s_{F_{b_{j}}}, \gamma_{b_{j}}\right)\right\rangle(j=1,2, \ldots, n)$ be two sets of 2 TLNNs. If $\Delta^{-1}\left(s_{T_{a_{j}}}, \alpha_{a_{j}}\right) \leq$ $\Delta^{-1}\left(s_{T_{b_{j}}}, \alpha_{b_{j}}\right), \Delta^{-1}\left(s_{I_{a_{j}}}, \beta_{a_{j}}\right) \geq \Delta^{-1}\left(s_{I_{b_{j}}}, \beta_{b_{j}}\right)$ and $\Delta^{-1}\left(s_{F_{a_{j}}}, \gamma_{a_{j}}\right) \geq \Delta^{-1}\left(s_{F_{b_{j}}}, \gamma_{b_{j}}\right)$ hold for all $j$, then

$$
2 \operatorname{TLNWDHM}^{(x)}\left(\varphi_{a_{1}}, \varphi_{a_{2}}, \cdots, \varphi_{a_{n}}\right) \leq 2 \operatorname{TLNWDHM}^{(x)}\left(\varphi_{b_{1}}, \varphi_{b_{2}}, \cdots, \varphi_{b_{n}}\right)
$$

Property 10. (Boundedness) Let $\varphi_{j}=\left\langle\left(s_{T_{j}}, \alpha_{j}\right),\left(s_{I^{\prime}}, \beta_{j}\right),\left(s_{F_{j}}, \gamma_{j}\right)\right\rangle(j=1,2, \ldots, n)$ be a set of 2 TLNNs. If $\varphi_{i}^{+}=\left(\max _{i}\left(s_{T_{j}}, \alpha_{j}\right), \min _{i}\left(s_{I_{j}}, \beta_{j}\right), \min _{i}\left(s_{F_{j}}, \gamma_{j}\right)\right)$ and $\varphi_{i}^{+}=$ $\left(\max _{i}\left(s_{T_{j}}, \alpha_{j}\right), \min _{i}\left(s_{I_{j}}, \beta_{j}\right), \min _{i}\left(s_{F_{j}}, \gamma_{j}\right)\right)$, then

$$
\varphi^{-} \leq 2 \operatorname{TLNWDHM}^{(x)}\left(\varphi_{1}, \varphi_{2}, \cdots, \varphi_{n}\right) \leq \varphi^{+}
$$

From Theorem 4,

$$
\begin{aligned}
& 2 \operatorname{TLNWDHM}^{(x)}\left(\varphi_{1}^{+}, \varphi_{2}^{+}, \cdots, \varphi_{n}^{+}\right) \\
& =\left\{\begin{array}{l}
\Delta\left(t\left(\prod_{1 \leq i_{1}<\ldots<i_{x} \leq n}\left(1-\left(\prod_{j=1}^{x}\left(1-\frac{\max \Delta^{-1}\left(s_{T_{j},}, \alpha_{j}\right)}{t}\right)^{w_{i_{j}}}\right)^{\frac{1}{x}}\right)\right)^{\frac{1}{C_{n}^{x}}}\right), \\
\left.\Delta\left(t\left(\prod_{1 \leq i_{1}<\ldots<i_{x} \leq n}\left(1-\left(\prod_{j=1}^{x}\left(\frac{\min \Delta^{-1}\left(s_{I_{j}}, \beta_{j}\right)}{t}\right)^{w_{i_{j}}}\right)^{\frac{1}{x}}\right)\right)^{\frac{1}{C_{n}^{x}}}\right)\right), \\
\Delta\left(t\left(1-\left(\prod_{1 \leq i_{1}<\ldots<i_{x} \leq n}\left(1-\left(\prod_{j=1}^{x}\left(\frac{\min \Delta^{-1}\left(s_{F_{j}}, \gamma_{j}\right)}{t}\right)^{w_{i_{j}}}\right)^{\frac{1}{x}}\right)\right)^{\frac{1}{C_{n}^{x}}}\right)\right.
\end{array}\right\}
\end{aligned}
$$

$$
2 \operatorname{TLNWDHM}^{(x)}\left(\varphi_{1}^{-}, \varphi_{2}^{-}, \cdots, \varphi_{n}^{-}\right)
$$

$$
=\left\{\begin{array}{l}
\Delta\left(t\left(\prod_{1 \leq i_{1}<\ldots<i_{x} \leq n}\left(1-\left(\prod_{j=1}^{x}\left(1-\frac{\min \Delta^{-1}\left(s_{T_{j},} \alpha_{j}\right)}{t}\right)^{w_{i_{j}}}\right)^{\frac{1}{x}}\right)\right)^{\frac{1}{C_{n}^{x}}}\right), \\
\left.\Delta\left(t-\left(\prod_{1 \leq i_{1}<\ldots<i_{x} \leq n}\left(1-\left(\prod_{j=1}^{x}\left(\frac{\max \Delta^{-1}\left(s_{I_{j}, \beta_{j}}\right)}{t}\right)^{w_{i_{j}}}\right)^{\frac{1}{x}}\right)\right)^{\frac{1}{C_{n}^{x}}}\right)\right), \\
\Delta\left(t\left(1-\left(\prod_{1 \leq i_{1}<\ldots<i_{x} \leq n}\left(1-\left(\prod_{j=1}^{x}\left(\frac{\max \Delta^{-1}\left(s_{F_{j},} \gamma_{j}\right)}{t}\right)^{w_{i_{j}}}\right)^{\frac{1}{x}}\right)\right)^{\frac{1}{C_{n}^{x}}}\right)\right)
\end{array}\right\}
$$

From Property 9,

$$
\varphi^{-} \leq 2 \operatorname{TLNWDHM}^{(x)}\left(\varphi_{1}, \varphi_{2}, \cdots, \varphi_{n}\right) \leq \varphi^{+}
$$

It is obvious that 2TLNWDHM operator lacks the property of idempotency. 


\section{Numerical Example and Comparative Analysis}

\subsection{Numerical Example}

The constructional engineering projects have the following characteristics: large investment, many participants, complex project environment, and a wide range of risk factors on the basis of the Engineering Procurement Construction (EPC) mode. Therefore, it is necessary to analyze and assess the constructional engineering project's risks during the life cycle; a risk assessment is good for implementing projects and completing project goals. During the process of implementation, constructional engineering projects face aspects of risk-political, economic, social and natural and other aspects of risks. These risks have a great influence on our construction company, and produce many factors with high probability that are difficult to estimate and quantify. Thus, we shall give a numerical example for construction engineering project risk assessment with 2TLNNs in order to illustrate the method proposed in this paper. There are five possible construction engineering projects $A_{i}(i=1,2,3,4,5)$ to evaluate. The expert selects four attributes to evaluate the five possible construction engineering projects: (1) $G_{1}$ is the construction work environment; (2) $G_{2}$ is the construction site safety protection measures; (3) $\mathrm{G}_{3}$ is the safety management ability of the engineering project management; and (4) $\mathrm{G}_{4}$ is the safety production responsibility system. The five possible construction engineering projects, $A_{i}(i=1,2,3,4,5)$, will be evaluated using the 2 TLNNs by the decision maker using the above four attributes (whose weighting vector is $\omega=(0.2,0.1,0.5,0.2)$ and expert weighting vector is $\omega=(0.2,0.4,0.4)$, which are listed in Tables $1-3$.

Table 1. 2-tuple linguistic neutrosophic numbers (2TLNN) decision matrix $\left(R_{1}\right)$.

\begin{tabular}{ccccc}
\hline & $\mathrm{G}_{1}$ & $\mathrm{G}_{2}$ & \multicolumn{1}{c}{$\mathrm{G}_{3}$} & $\mathrm{G}_{4}$ \\
\hline $\mathrm{A}_{1}$ & $<\left(\mathrm{s}_{4}, 0\right),\left(\mathrm{s}_{3}, 0\right)\left(\mathrm{s}_{2}, 0\right)>$ & $<\left(\mathrm{s}_{5}, 0\right),\left(\mathrm{s}_{3}, 0\right)\left(\mathrm{s}_{1}, 0\right)>$ & $<\left(\mathrm{s}_{4}, 0\right),\left(\mathrm{s}_{1}, 0\right)\left(\mathrm{s}_{2}, 0\right)>$ & $<\left(\mathrm{s}_{2}, 0\right),\left(\mathrm{s}_{3}, 0\right)\left(\mathrm{s}_{2}, 0\right)>$ \\
$\mathrm{A}_{2}$ & $<\left(\mathrm{s}_{3}, 0\right),\left(\mathrm{s}_{2}, 0\right)\left(\mathrm{s}_{4}, 0\right)>$ & $<\left(\mathrm{s}_{4}, 0\right),\left(\mathrm{s}_{2}, 0\right)\left(\mathrm{s}_{2}, 0\right)>$ & $<\left(\mathrm{s}_{3}, 0\right),\left(\mathrm{s}_{2}, 0\right)\left(\mathrm{s}_{2}, 0\right)>$ & $<\left(\mathrm{s}_{4}, 0\right),\left(\mathrm{s}_{3}, 0\right)\left(\mathrm{s}_{3}, 0\right)>$ \\
$\mathrm{A}_{3}$ & $<\left(\mathrm{s}_{5}, 0\right),\left(\mathrm{s}_{4}, 0\right)\left(\mathrm{s}_{3}, 0\right)>$ & $<\left(\mathrm{s}_{4}, 0\right),\left(\mathrm{s}_{4}, 0\right)\left(\mathrm{s}_{3}, 0\right)>$ & $<\left(\mathrm{s}_{2}, 0\right),\left(\mathrm{s}_{1}, 0\right)\left(\mathrm{s}_{2}, 0\right)>$ & $<\left(\mathrm{s}_{4}, 0\right),\left(\mathrm{s}_{3}, 0\right)\left(\mathrm{s}_{2}, 0\right)>$ \\
$\mathrm{A}_{4}$ & $<\left(\mathrm{s}_{2}, 0\right),\left(\mathrm{s}_{1}, 0\right)\left(\mathrm{s}_{2}, 0\right)>$ & $<\left(\mathrm{s}_{5}, 0\right),\left(\mathrm{s}_{1}, 0\right)\left(\mathrm{s}_{2}, 0\right)>$ & $<\left(\mathrm{s}_{4}, 0\right),\left(\mathrm{s}_{3}, 0\right)\left(\mathrm{s}_{5}, 0\right)>$ & $<\left(\mathrm{s}_{3}, 0\right),\left(\mathrm{s}_{1}, 0\right)\left(\mathrm{s}_{1}, 0\right)>$ \\
$\mathrm{A}_{5}$ & $<\left(\mathrm{s}_{4}, 0\right),\left(\mathrm{s}_{3}, 0\right)\left(\mathrm{s}_{1}, 0\right)>$ & $<\left(\mathrm{s}_{5}, 0\right),\left(\mathrm{s}_{2}, 0\right)\left(\mathrm{s}_{2}, 0\right)>$ & $<\left(\mathrm{s}_{3}, 0\right),\left(\mathrm{s}_{2}, 0\right)\left(\mathrm{s}_{1}, 0\right)>$ & $<\left(\mathrm{s}_{3}, 0\right),\left(\mathrm{s}_{2}, 0\right)\left(\mathrm{s}_{2}, 0\right)>$ \\
\hline
\end{tabular}

Table 2. 2TLNN decision matrix $\left(R_{2}\right)$.

\begin{tabular}{cccccc}
\hline & $\mathrm{G}_{\mathbf{1}}$ & $\mathrm{G}_{\mathbf{2}}$ & \multicolumn{1}{c}{$\mathrm{G}_{3}$} & $\mathrm{G}_{4}$ \\
\hline $\mathrm{A}_{1}$ & $<\left(\mathrm{s}_{3}, 0\right),\left(\mathrm{s}_{2}, 0\right)\left(\mathrm{s}_{3}, 0\right)>$ & $<\left(\mathrm{s}_{3}, 0\right),\left(\mathrm{s}_{3}, 0\right)\left(\mathrm{s}_{2}, 0\right)>$ & $<\left(\mathrm{s}_{3}, 0\right),\left(\mathrm{s}_{1}, 0\right)\left(\mathrm{s}_{2}, 0\right)>$ & $<\left(\mathrm{s}_{4}, 0\right),\left(\mathrm{s}_{1}, 0\right)\left(\mathrm{s}_{3}, 0\right)>$ \\
$\mathrm{A}_{2}$ & $<\left(\mathrm{s}_{2}, 0\right),\left(\mathrm{s}_{3}, 0\right)\left(\mathrm{s}_{3}, 0\right)>$ & $<\left(\mathrm{s}_{3}, 0\right),\left(\mathrm{s}_{3}, 0\right)\left(\mathrm{s}_{3}, 0\right)>$ & $<\left(\mathrm{s}_{3}, 0\right),\left(\mathrm{s}_{2}, 0\right)\left(\mathrm{s}_{2}, 0\right)>$ & $<\left(\mathrm{s}_{3}, 0\right),\left(\mathrm{s}_{4}, 0\right)\left(\mathrm{s}_{3}, 0\right)>$ \\
$\mathrm{A}_{3}$ & $<\left(\mathrm{s}_{2}, 0\right),\left(\mathrm{s}_{3}, 0\right)\left(\mathrm{s}_{3}, 0\right)>$ & $<\left(\mathrm{s}_{3}, 0\right),\left(\mathrm{s}_{2}, 0\right)\left(\mathrm{s}_{2}, 0\right)>$ & $<\left(\mathrm{s}_{2}, 0\right),\left(\mathrm{s}_{3}, 0\right)\left(\mathrm{s}_{1}, 0\right)>$ & $<\left(\mathrm{s}_{3}, 0\right),\left(\mathrm{s}_{2}, 0\right)\left(\mathrm{s}_{4}, 0\right)>$ \\
$\mathrm{A}_{4}$ & $<\left(\mathrm{s}_{3}, 0\right),\left(\mathrm{s}_{2}, 0\right)\left(\mathrm{s}_{2}, 0\right)>$ & $<\left(\mathrm{s}_{2}, 0\right),\left(\mathrm{s}_{2}, 0\right)\left(\mathrm{s}_{3}, 0\right)>$ & $<\left(\mathrm{s}_{3}, 0\right),\left(\mathrm{s}_{4}, 0\right)\left(\mathrm{s}_{2}, 0\right)>$ & $<\left(\mathrm{s}_{3}, 0\right),\left(\mathrm{s}_{1}, 0\right)\left(\mathrm{s}_{2}, 0\right)>$ \\
$\mathrm{A}_{5}$ & $<\left(\mathrm{s}_{3}, 0\right),\left(\mathrm{s}_{2}, 0\right)\left(\mathrm{s}_{1}, 0\right)>$ & $<\left(\mathrm{s}_{3}, 0\right),\left(\mathrm{s}_{4}, 0\right)\left(\mathrm{s}_{3}, 0\right)>$ & $<\left(\mathrm{s}_{4}, 0\right),\left(\mathrm{s}_{1}, 0\right)\left(\mathrm{s}_{1}, 0\right)>$ & $<\left(\mathrm{s}_{2}, 0\right),\left(\mathrm{s}_{3}, 0\right)\left(\mathrm{s}_{2}, 0\right)>$ \\
\hline
\end{tabular}

Table 3. 2TLNN decision matrix $\left(R_{3}\right)$.

\begin{tabular}{cccccc}
\hline & \multicolumn{1}{c}{$\mathrm{G}_{\mathbf{1}}$} & $\mathrm{G}_{\mathbf{2}}$ & \multicolumn{1}{c}{$\mathrm{G}_{3}$} & $\mathbf{G}_{4}$ \\
\hline $\mathrm{A}_{1}$ & $<\left(\mathrm{s}_{3}, 0\right),\left(\mathrm{s}_{3}, 0\right)\left(\mathrm{s}_{1}, 0\right)>$ & $<\left(\mathrm{s}_{4}, 0\right),\left(\mathrm{s}_{2}, 0\right)\left(\mathrm{s}_{1}, 0\right)>$ & $<\left(\mathrm{s}_{4}, 0\right),\left(\mathrm{s}_{4}, 0\right)\left(\mathrm{s}_{3}, 0\right)>$ & $<\left(\mathrm{s}_{4}, 0\right),\left(\mathrm{s}_{1}, 0\right)\left(\mathrm{s}_{3}, 0\right)>$ \\
$\mathrm{A}_{2}$ & $<\left(\mathrm{s}_{2}, 0\right),\left(\mathrm{s}_{2}, 0\right)\left(\mathrm{s}_{2}, 0\right)>$ & $<\left(\mathrm{s}_{4}, 0\right),\left(\mathrm{s}_{4}, 0\right)\left(\mathrm{s}_{4}, 0\right)>$ & $<\left(\mathrm{s}_{3}, 0\right),\left(\mathrm{s}_{2}, 0\right)\left(\mathrm{s}_{3}, 0\right)>$ & $<\left(\mathrm{s}_{2}, 0\right),\left(\mathrm{s}_{1}, 0\right)\left(\mathrm{s}_{3}, 0\right)>$ \\
$\mathrm{A}_{3}$ & $<\left(\mathrm{s}_{2}, 0\right),\left(\mathrm{s}_{1}, 0\right)\left(\mathrm{s}_{2}, 0\right)>$ & $<\left(\mathrm{s}_{3}, 0\right),\left(\mathrm{s}_{2}, 0\right)\left(\mathrm{s}_{2}, 0\right)>$ & $<\left(\mathrm{s}_{4}, 0\right),\left(\mathrm{s}_{5}, 0\right)\left(\mathrm{s}_{2}, 0\right)>$ & $<\left(\mathrm{s}_{2}, 0\right),\left(\mathrm{s}_{4}, 0\right)\left(\mathrm{s}_{4}, 0\right)>$ \\
$\mathrm{A}_{4}$ & $<\left(\mathrm{s}_{3}, 0\right),\left(\mathrm{s}_{1}, 0\right)\left(\mathrm{s}_{2}, 0\right)>$ & $<\left(\mathrm{s}_{2}, 0\right),\left(\mathrm{s}_{1}, 0\right)\left(\mathrm{s}_{2}, 0\right)>$ & $<\left(\mathrm{s}_{3}, 0\right),\left(\mathrm{s}_{4}, 0\right)\left(\mathrm{s}_{5}, 0\right)>$ & $<\left(\mathrm{s}_{5}, 0\right),\left(\mathrm{s}_{3}, 0\right)\left(\mathrm{s}_{1}, 0\right)>$ \\
$\mathrm{A}_{5}$ & $<\left(\mathrm{s}_{3}, 0\right),\left(\mathrm{s}_{3}, 0\right)\left(\mathrm{s}_{2}, 0\right)>$ & $<\left(\mathrm{s}_{3}, 0\right),\left(\mathrm{s}_{2}, 0\right)\left(\mathrm{s}_{2}, 0\right)>$ & $<\left(\mathrm{s}_{3}, 0\right),\left(\mathrm{s}_{2}, 0\right)\left(\mathrm{s}_{3}, 0\right)>$ & $<\left(\mathrm{s}_{5}, 0\right),\left(\mathrm{s}_{3}, 0\right)\left(\mathrm{s}_{4}, 0\right)>$ \\
\hline
\end{tabular}

Then, we utilize the approach developed to select the best construction engineering projects. 
Definition 12. Let $\varphi_{j}=\left\langle\left(s_{T_{j}}, \alpha_{j}\right),\left(s_{I_{j}}, \beta_{j}\right),\left(s_{F_{j}}, \gamma_{j}\right)\right\rangle(j=1,2, \ldots, n)$ be a set of $2 T L N N s$ with the weight vector, $w_{i}=\left(w_{1}, w_{2}, \ldots, w_{n}\right)^{T}$, thereby satisfying $w_{i} \in[0,1]$ and $\sum_{i=1}^{n} w_{i}=1$, then we can obtain

$$
\begin{aligned}
& \quad \begin{array}{l}
\operatorname{TTLNNWAA}\left(\varphi_{1}, \varphi_{2}, \ldots, \varphi_{n}\right)=\sum_{j=1}^{n} w_{j} \varphi_{j} \\
=\left\{\begin{array}{l}
\left.\Delta\left(1-\prod_{j=1}^{n}\left(1-\frac{\Delta^{-1}\left(s_{T_{j}}, \alpha_{j}\right)}{t}\right)^{w_{j}}\right)\right), \\
\left.\Delta\left(t\left(\prod_{j=1}^{n}\left(\frac{\Delta^{-1}\left(s_{I_{j}}, \beta_{j}\right)}{t}\right)^{w_{j}}\right)\right), \Delta\left(t\left(\prod_{j=1}^{n}\left(\frac{\Delta^{-1}\left(s_{F_{j}}, \gamma_{j}\right)}{t}\right)\right)^{w_{j}}\right)\right)
\end{array}\right\} \\
2 \operatorname{TLNNWGA}\left(\varphi_{1}, \varphi_{2}, \ldots, \varphi_{n}\right)=\sum_{j=1}^{n}\left(\varphi_{j}\right)^{w_{j}} \\
=\left\{\begin{array}{l}
\Delta\left(t\left(\prod_{j=1}^{n}\left(\frac{\Delta^{-1}\left(s_{T_{j},}, \alpha_{j}\right)}{t}\right)^{w_{j}}\right)\right), \Delta\left(t\left(1-\prod_{j=1}^{n}\left(1-\frac{\Delta^{-1}\left(s_{I_{j}}, \beta_{j}\right)}{t}\right)^{w_{j}}\right)\right), \\
\Delta\left(t\left(1-\prod_{j=1}^{n}\left(1-\frac{\Delta^{-1}\left(s_{F_{j}}, \gamma_{j}\right)}{t}\right)^{w_{j}}\right)\right)
\end{array}\right\}
\end{array}
\end{aligned}
$$

Step 1. According to the 2TLNNs, $r_{i j}(i=1,2,3,4,5, j=1,2,3,4)$, we can calculate all 2TLNNs $r_{i j}$ by using the 2-tuple linguistic neutrosophic numbers weighted average (2TLNNWA) operator and 2-tuple linguistic neutrosophic numbers weighted geometric (2TLNNWG) operator to get the overall 2 TLNNs $A_{i}(i=1,2,3,4,5)$ of the construction engineering projects. Then, the results are shown in Table 4.

Table 4. The aggregating results by the 2TLNNWAA operator.

\begin{tabular}{ccc}
\hline & $\mathrm{G}_{1}$ & $\mathrm{G}_{2}$ \\
\hline $\mathrm{A}_{1}$ & $<\left(\mathrm{s}_{3}, 0.2337\right),\left(\mathrm{s}_{3},-0.4492\right),\left(\mathrm{s}_{2},-0.2174\right)>$ & $<\left(\mathrm{s}_{4},-0.0477\right),\left(\mathrm{s}_{3},-0.4492\right),\left(\mathrm{s}_{1}, 0.3195\right)>$ \\
$\mathrm{A}_{2}$ & $<\left(\mathrm{s}_{2}, 0.2236\right),\left(\mathrm{s}_{2}, 0.3522\right),\left(\mathrm{s}_{3},-0.2981\right)>$ & $<\left(\mathrm{s}_{4},-0.3522\right),\left(\mathrm{s}_{3}, 0.1037\right),\left(\mathrm{s}_{3}, 0.1037\right)>$ \\
$\mathrm{A}_{3}$ & $<\left(\mathrm{s}_{3},-0.0314\right),\left(\mathrm{s}_{2}, 0.0477\right),\left(\mathrm{s}_{3},-0.4492\right)>$ & $<\left(\mathrm{s}_{3}, 0.2337\right),\left(\mathrm{s}_{2}, 0.2974\right),\left(\mathrm{s}_{2}, 0.1689\right)>$ \\
$\mathrm{A}_{4}$ & $<\left(\mathrm{s}_{3},-0.1777\right),\left(\mathrm{s}_{1}, 0.3195\right),\left(\mathrm{s}_{2}, 0.0000\right)>$ & $<\left(\mathrm{s}_{3},-0.0314\right),\left(\mathrm{s}_{1}, 0.3195\right),\left(\mathrm{s}_{2}, 0.3522\right)>$ \\
$\mathrm{A}_{5}$ & $<\left(\mathrm{s}_{3}, 0.2337\right),\left(\mathrm{s}_{3},-0.4492\right),\left(\mathrm{s}_{1}, 0.3195\right)>$ & $<\left(\mathrm{s}_{4},-0.4082\right),\left(\mathrm{s}_{3},-0.3610\right),\left(\mathrm{s}_{2}, 0.3522\right)>$ \\
\hline \multicolumn{4}{c}{$\mathrm{G}_{3}$} & $\mathrm{G}_{4}$ \\
\hline $\mathrm{A}_{1}$ & $<\left(\mathrm{s}_{4},-0.3522\right),\left(\mathrm{s}_{2},-0.2589\right),\left(\mathrm{s}_{2}, 0.3522\right)>$ & $<\left(\mathrm{s}_{4},-0.2974\right),\left(\mathrm{s}_{1}, 0.2457\right),\left(\mathrm{s}_{3},-0.2337\right)>$ \\
$\mathrm{A}_{2}$ & $<\left(\mathrm{s}_{3}, 0.000\right),\left(\mathrm{s}_{2}, 0.0000\right),\left(\mathrm{s}_{2}, 0.3522\right)>$ & $<\left(\mathrm{s}_{3},-0.1037\right),\left(\mathrm{s}_{2}, 0.1689\right),\left(\mathrm{s}_{3}, 0.0000\right)>$ \\
$\mathrm{A}_{3}$ & $<\left(\mathrm{s}_{3},-0.0314\right),\left(\mathrm{s}_{3},-0.0458\right),\left(\mathrm{s}_{2},-0.4843\right)>$ & $<\left(\mathrm{s}_{3},-0.1037\right),\left(\mathrm{s}_{3},-0.1381\right),\left(\mathrm{s}_{3}, 0.4822\right)>$ \\
$\mathrm{A}_{4}$ & $<\left(\mathrm{s}_{3}, 0.2337\right),\left(\mathrm{s}_{4},-0.2236\right),\left(\mathrm{s}_{3}, 0.4657\right)>$ & $<\left(\mathrm{s}_{4}, 0.0668\right),\left(\mathrm{s}_{2},-0.4482\right),\left(\mathrm{s}_{1}, 0.3195\right)>$ \\
$\mathrm{A}_{5}$ & $<\left(\mathrm{s}_{3}, 0.4492\right),\left(\mathrm{s}_{2},-0.4843\right),\left(\mathrm{s}_{2},-0.4482\right)>$ & $<\left(\mathrm{s}_{4},-0.1689\right),\left(\mathrm{s}_{3},-0.2337\right),\left(\mathrm{s}_{3},-0.3610\right)>$ \\
\hline
\end{tabular}

Step 2. According to Table 4, we can calculate the $r_{i j}$ of all 2TLNNs by using the 2TLNWHM (2TLNWDHM) operator to get the overall 2TLNNs $A_{i}(i=1,2,3,4,5)$ of the construction engineering projects, $A_{i}$. Suppose that $x=2$, then the aggregating results are as shown in Table 5 .

Table 5. The aggregating results of the construction engineering projects by the 2TLNWHM (2TLNWDHM) operator.

\begin{tabular}{ccc}
\hline & 2TLNWHM & 2TLNWDHM \\
\hline $\mathrm{A}_{1}$ & $<\left(\mathrm{s}_{5}, 0.3257\right),\left(\mathrm{s}_{1},-0.4776\right),\left(\mathrm{s}_{1},-0.4012\right)>$ & $<\left(\mathrm{s}_{1}, 0.1564\right),\left(\mathrm{s}_{5},-0.4119\right),\left(\mathrm{s}_{5},-0.2877\right)>$ \\
$\mathrm{A}_{2}$ & $<\left(\mathrm{s}_{5}, 0.0468\right),\left(\mathrm{s}_{1},-0.3632\right),\left(\mathrm{s}_{1},-0.2177\right)>$ & $<\left(\mathrm{s}_{1},-0.1456\right),\left(\mathrm{s}_{5},-0.2124\right),\left(\mathrm{s}_{5},-0.0171\right)>$ \\
$\mathrm{A}_{3}$ & $<\left(\mathrm{s}_{5}, 0.1002\right),\left(\mathrm{s}_{1},-0.2491\right),\left(\mathrm{s}_{1},-0.3474\right)>$ & $<\left(\mathrm{s}_{1},-0.1092\right),\left(\mathrm{s}_{5},-0.0603\right),\left(\mathrm{s}_{5},-0.1946\right)>$ \\
$\mathrm{A}_{4}$ & $<\left(\mathrm{s}_{5}, 0.2143\right),\left(\mathrm{s}_{1},-0.3927\right),\left(\mathrm{s}_{1},-0.3263\right)>$ & $<\left(\mathrm{s}_{1}, 0.0275\right),\left(\mathrm{s}_{5},-0.2639\right),\left(\mathrm{s}_{5},-0.1713\right)>$ \\
$\mathrm{A}_{5}$ & $<\left(\mathrm{s}_{5}, 0.2941\right),\left(\mathrm{s}_{1},-0.3870\right),\left(\mathrm{s}_{1},-0.4981\right)>$ & $<\left(\mathrm{s}_{1},-0.1127\right),\left(\mathrm{s}_{5},-0.2367\right),\left(\mathrm{s}_{5},-0.4415\right)>$ \\
\hline
\end{tabular}


Step 3. In accordance with the aggregating results shown in Table 5, the score functions of the construction engineering projects are shown in Table 6.

Table 6. The score functions of the construction engineering projects.

\begin{tabular}{ccc}
\hline & 2TLNWHM & 2TLNWDHM \\
\hline $\mathrm{A}_{1}$ & $\left(\mathrm{~s}_{5}, 0.4015\right)$ & $\left(\mathrm{s}_{1}, 0.2853\right)$ \\
$\mathrm{A}_{2}$ & $\left(\mathrm{~s}_{5}, 0.2092\right)$ & $\left(\mathrm{s}_{1}, 0.0280\right)$ \\
$\mathrm{A}_{3}$ & $\left(\mathrm{~s}_{5}, 0.2323\right)$ & $\left(\mathrm{s}_{1}, 0.0486\right)$ \\
$\mathrm{A}_{4}$ & $\left(\mathrm{~s}_{5}, 0.3111\right)$ & $\left(\mathrm{s}_{1}, 0.1542\right)$ \\
$\mathrm{A}_{5}$ & $\left(\mathrm{~s}_{5}, 0.3930\right)$ & $\left(\mathrm{s}_{1}, 0.2636\right)$ \\
\hline
\end{tabular}

Step 4. In accordance with the scores shown in Table 6 and the comparison formulas of the score functions, the ordering of the construction engineering projects are shown in Table 7 . The best construction engineering project is $\mathrm{A}_{1}$.

Table 7. Ordering of the construction engineering projects.

\begin{tabular}{cc}
\hline & Ordering \\
\hline 2TLNWHM & $\mathrm{A}_{1}>\mathrm{A}_{5}>\mathrm{A}_{4}>\mathrm{A}_{3}>\mathrm{A}_{2}$ \\
2TLNWDHM & $\mathrm{A}_{1}>\mathrm{A}_{5}>\mathrm{A}_{4}>\mathrm{A}_{3}>\mathrm{A}_{2}$ \\
\hline
\end{tabular}

\subsection{Influence of the Parameter on the Final Result}

In order to show the effects on the ranking results by changing parameters of $x$ in the 2TLNWHM (2TLNWDHM) operators, all the results are shown in Tables 8 and 9.

Table 8. Ranking results for different operational parameters of the 2TLNWHM operator.

\begin{tabular}{ccccccc}
\hline & $\mathbf{s}\left(\mathbf{A}_{\mathbf{1}}\right)$ & $\mathbf{s}\left(\mathbf{A}_{\mathbf{2}}\right)$ & $\mathbf{s}\left(\mathbf{A}_{\mathbf{3}}\right)$ & $\mathbf{s}\left(\mathbf{A}_{\mathbf{4}}\right)$ & $\mathbf{s}\left(\mathbf{A}_{\mathbf{5}}\right)$ & Ordering \\
\hline$x=1$ & 0.9134 & 0.8799 & 0.8865 & 0.9070 & 0.9065 & $\mathrm{~A}_{1}>\mathrm{A}_{4}>\mathrm{A}_{5}>\mathrm{A}_{3}>\mathrm{A}_{2}$ \\
$x=2$ & 0.9003 & 0.8682 & 0.8720 & 0.8852 & 0.8988 & $\mathrm{~A}_{1}>\mathrm{A}_{5}>\mathrm{A}_{4}>\mathrm{A}_{3}>\mathrm{A}_{2}$ \\
$x=3$ & 0.8953 & 0.8642 & 0.8661 & 0.8696 & 0.8958 & $\mathrm{~A}_{5}>\mathrm{A}_{1}>\mathrm{A}_{4}>\mathrm{A}_{3}>\mathrm{A}_{2}$ \\
$x=4$ & 0.8927 & 0.8621 & 0.8627 & 0.8571 & 0.8942 & $\mathrm{~A}_{5}>\mathrm{A}_{1}>\mathrm{A}_{2}>\mathrm{A}_{3}>\mathrm{A}_{4}$ \\
\hline
\end{tabular}

Table 9. Ranking results for different operational parameters of the 2TLNWDHM operator.

\begin{tabular}{ccccccc}
\hline & $\mathbf{s}\left(\mathbf{A}_{\mathbf{1}}\right)$ & $\mathbf{s}\left(\mathbf{A}_{\mathbf{2}}\right)$ & $\mathbf{s}\left(\mathbf{A}_{\mathbf{3}}\right)$ & $\mathbf{s}\left(\mathbf{A}_{\mathbf{4}}\right)$ & $\mathbf{s}\left(\mathbf{A}_{5}\right)$ & Ordering \\
\hline$x=1$ & 0.1922 & 0.1489 & 0.1557 & 0.1796 & 0.1817 & $\mathrm{~A}_{1}>\mathrm{A}_{5}>\mathrm{A}_{4}>\mathrm{A}_{3}>\mathrm{A}_{2}$ \\
$x=2$ & 0.2142 & 0.1713 & 0.1748 & 0.1924 & 0.2106 & $\mathrm{~A}_{1}>\mathrm{A}_{5}>\mathrm{A}_{4}>\mathrm{A}_{3}>\mathrm{A}_{2}$ \\
$x=3$ & 0.2239 & 0.1815 & 0.1852 & 0.1965 & 0.2252 & $\mathrm{~A}_{5}>\mathrm{A}_{1}>\mathrm{A}_{4}>\mathrm{A}_{3}>\mathrm{A}_{2}$ \\
$x=4$ & 0.2293 & 0.1878 & 0.1922 & 0.1985 & 0.2342 & $\mathrm{~A}_{5}>\mathrm{A}_{1}>\mathrm{A}_{4}>\mathrm{A}_{3}>\mathrm{A}_{2}$ \\
\hline
\end{tabular}

\subsection{Comparative Analysis}

Then, we compare our proposed method with the LNNWAA operator and LNNWGA operator [33] and cosine measures of linguistic neutrosophic numbers [34]. The comparative results are shown in Table 10.

Table 10. Ordering of the construction engineering projects.

\begin{tabular}{cc}
\hline & Ordering \\
\hline LNNWAA [33] & $\mathrm{A}_{5}>\mathrm{A}_{1}>\mathrm{A}_{4}>\mathrm{A}_{3}>\mathrm{A}_{2}$ \\
LNNWGA [33] & $\mathrm{A}_{5}>\mathrm{A}_{1}>\mathrm{A}_{3}>\mathrm{A}_{2}>\mathrm{A}_{4}$ \\
$C^{w_{1}}$ LNNs [34] & $\mathrm{A}_{5}>\mathrm{A}_{1}>\mathrm{A}_{4}>\mathrm{A}_{2}>\mathrm{A}_{3}$ \\
$C^{w_{2}}$ LNNs [34] & $\mathrm{A}_{5}>\mathrm{A}_{1}>\mathrm{A}_{4}>\mathrm{A}_{2}>\mathrm{A}_{3}$ \\
\hline
\end{tabular}


From above, we determine that the optimal construction engineering projects to show the practicality and effectiveness of the proposed approaches. However, the LNNWAA operator and LNNWGA operator do not consider the information about the relationship between the arguments being aggregated and thus, cannot eliminate the influence of unfair arguments on the decision result. Our proposed 2TLNWHM and 2TLNWDHM operators consider the information about the relationship among arguments being aggregated.

\section{Conclusions}

In this paper, we investigated the MADM problems with 2TLNNs. Then, we utilized the Hamy mean (HM) operator, weighted Hamy mean (WHM) operator, dual Hamy mean (DHM) operator and weighted dual Hamy mean (WDHM) operator to develop some Hamy mean aggregation operators with 2TLNNs: 2-tuple linguistic neutrosophic Hamy mean (2TLNHM) operator, 2-tuple linguistic neutrosophic weighted Hamy mean (2TLNWHM) operator, 2-tuple linguistic neutrosophic dual Hamy mean (2TLNDHM) operator, and 2-tuple linguistic neutrosophic weighted dual Hamy mean (2TLNWDHM) operator. The prominent characteristics of these proposed operators were studied. Then, we utilized these operators to develop some approaches to solve MADM problems with 2TLNNs. Finally, a practical example for construction engineering project risk assessment was given to show the developed approach. In the future, the application of the 2TLNNs needs to be investigated under uncertain [35-46] and fuzzy environments [47-54].

Author Contributions: S.W., J.W., G.W. and Y.W. conceived and worked together to achieve this work, J.W. compiled the computing program by Matlab and analyzed the data, J.W. and G.W. wrote the paper. Finally, all the authors have read and approved the final manuscript.

Acknowledgments: The work was supported by the National Natural Science Foundation of China under Grant No. 71571128 and the Humanities and Social Sciences Foundation of Ministry of Education of the People's Republic of China (16XJA630005) and the Construction Plan of Scientific Research Innovation Team for Colleges and Universities in Sichuan Province (15TD0004).

Conflicts of Interest: The authors declare no conflict of interest.

\section{References}

1. Smarandache, F. Neutrosophy: Neutrosophic Probability, Set, and Logic: Analytic Synthesis E Synthetic Analysis; American Research Press: Rehoboth, DE, USA, 1998.

2. Smarandache, F. A Unifying Field in Logics: Neutrosophic Logic. Neutrosophy, Neutrosophic Set, Neutrosophic Probability and Statistics, 3rd ed.; Phoenix: Xiquan, China, 2003.

3. Zadeh, L.A. Fuzzy sets. Inf. Control 1965, 8, 338-353. [CrossRef]

4. Atanassov, K. Intuitionistic fuzzy sets. Fuzzy Sets Syst. 1986, 20, 87-96. [CrossRef]

5. Atanassov, K.; Gargov, G. Interval-valued intuitionistic fuzzy sets. Fuzzy Sets Syst. 1989, 31, 343-349. [CrossRef]

6. Wang, H.; Smarandache, F.; Zhang, Y.Q.; Sunderraman, R. Single valued neutrosophic sets. Multispace Multistruct. 2010, 4, 410-413.

7. Wang, H.; Smarandache, F.; Zhang, Y.Q.; Sunderraman, R. Interval Neutrosophic Sets and Logic: Theory and Applications in Computing; Hexis: Phoenix, AZ, USA, 2005.

8. Ye, J. Multicriteria decision-making method using the correlation coefficient under single-valued neutrosophic environment. Int. J. General Syst. 2013, 42, 386-394. [CrossRef]

9. Broumi, S.; Smarandache, F. Correlation coefficient of interval neutrosophic set. Appl. Mech. Mater. 2013, 436, 511-517. [CrossRef]

10. Biswas, P.; Pramanik, S.; Giri, B.C. TOPSIS method for multi-attribute group decision-making under single-valued neutrosophic environment. Neural Comput. Appl. 2016, 27, 727-737. [CrossRef]

11. Liu, P.D.; Chu, Y.C.; Li, Y.W.; Chen, Y.B. Some generalized neutrosophic number Hamacher aggregation operators and their application to Group Decision Making. Int. J. Fuzzy Syst. 2014, 16, 242-255.

12. Sahin, R.; Liu, P.D. Maximizing deviation method for neutrosophic multiple attribute decision making with incomplete weight information. Neural Comput. Appl. 2016, 27, 2017-2029. [CrossRef] 
13. Ye, J. Similarity measures between interval neutrosophic sets and their applications in multicriteria decision-making. J. Intell. Fuzzy Syst. 2014, 26, 165-172.

14. Zhang, H.Y.; Wang, J.Q.; Chen, X.H. Interval neutrosophic sets and their application in multicriteria decision making problems. Sci. Word J. 2014, 2014, 1-15. [CrossRef] [PubMed]

15. Ye, J. A multicriteria decision-making method using aggregation operators for simplified neutrosophic sets. J. Intell. Fuzzy Syst. 2014, 26, 2459-2466.

16. Peng, J.J.; Wang, J.Q.; Wang, J.; Zhang, H.Y.; Chen, X.H. Simplified neutrosophic sets and their applications in multicriteria group decision-making problems. Int. J. Syst. Sci. 2016, 47, 2342-2358. [CrossRef]

17. Peng, J.J.; Wang, J.Q.; Zhang, H.Y.; Chen, X.H. An outranking approach for multi-criteria decision-making problems with simplified neutrosophic sets. Appl. Soft Comput. 2014, 25, 336-346. [CrossRef]

18. Zhang, H.; Wang, J.Q.; Chen, X.H. An outranking approach for multi-criteria decision-making problems with interval-valued neutrosophic sets. Neural Comput. Appl. 2016, 27, 615-627. [CrossRef]

19. Liu, P.D.; Xi, L. The neutrosophic number generalized weighted power averaging operator and its application in multiple attribute group decision making. Int. J. Mach. Learn. Cybernet. 2016. [CrossRef]

20. Deli, I.; Şubaş, Y. A ranking method of single valued neutrosophic numbers and its applications to multiattribute decision making problem. Int. J. Mach. Learn. Cybernet. 2017, 8, 1309-1322. [CrossRef]

21. Peng, J.J.; Wang, J.Q.; Wu, X.H.; Wang, J.; Chen, X.H. Multi-valued neutrosophic sets and power aggregation operators with their applications in multi-criteria group decision-making problems. Int. J. Comput. Intell. Syst. 2015, 8, 345-363. [CrossRef]

22. Zhang, H.Y.; Ji, P.; Wang, J.Q.; Chen, X.H. An improved weighted correlation coefficient based on integrated weight for interval neutrosophic sets and its application in multi-criteria decision-making problems. Int. J. Comput. Intell. Syst. 2015, 8, 1027-1043. [CrossRef]

23. Chen, J.Q.; Ye, J. Some Single-Valued Neutrosophic Dombi Weighted Aggregation Operators for Multiple Attribute Decision-Making. Symmetry 2017, 9, 82. [CrossRef]

24. Liu, P.D.; Wang, Y.M. Multiple attribute decision making method based on single-valued neutrosophic normalized weighted Bonferroni mean. Neural Comput. Appl. 2014, 25, 2001-2010. [CrossRef]

25. Wu, X.H.; Wang, J.Q.; Peng, J.J.; Chen, X.H. Cross-entropy and prioritized aggregation operator with simplified neutrosophic sets and their application in multi-criteria decision-making problems. J. Intell. Fuzzy Syst. 2016, 18, 1104-1116. [CrossRef]

26. Li, Y.; Liu, P.; Chen, Y. Some Single Valued Neutrosophic Number Heronian Mean Operators and Their Application in Multiple Attribute Group Decision Making. Informatica 2016, 27, 85-110. [CrossRef]

27. Zavadskas, E.K.; Bausys, R.; Juodagalviene, B.; Garnyte-Sapranaviciene, I. Model for residential house element and material selection by neutrosophic MULTIMOORA method. Eng. Appl. Artif. Intell. 2017, 64, 315-324. [CrossRef]

28. Zavadskas, E.K.; Bausys, R.; Kaklauskas, A.; Ubarte, I.; Kuzminske, A.; Gudiene, N. Sustainable market valuation of buildings by the single-valued neutrosophic MAMVA method. Appl. Soft Comput. 2017, 57, 74-87. [CrossRef]

29. Bausys, R.; Juodagalviene, B. Garage location selection for residential house by WASPAS-SVNS method. J. Civ. Eng. Manag. 2017, 23, 421-429. [CrossRef]

30. Wu, Q.; Wu, P.; Zhou, L.G.; Chen, H.Y.; Guan, X.J. Some new Hamacher aggregation operators under single-valued neutrosophic 2-tuple linguistic environment and their applications to multi-attribute group decision making. Comput. Ind. Eng. 2017, 116. [CrossRef]

31. Herrera, F.; Martinez, L. A 2-tuple fuzzy linguistic representation model for computing with words. IEEE Trans. Fuzzy Syst. 2000, 8, 746-752.

32. Hara, T.; Uchiyama, M.; Takahasi, S.E. A refinement of various mean inequalities. J. Inequal. Appl. 1998, 2, 387-395. [CrossRef]

33. Fang, Z.; Ye, J. Multiple Attribute Group Decision-Making Method Based on Linguistic Neutrosophic Numbers. Symmetry 2017, 9, 111. [CrossRef]

34. Shi, L.; Ye, J. Cosine Measures of Linguistic Neutrosophic Numbers and Their Application in Multiple Attribute Group Decision-Making. Information 2017, 8, 117.

35. Chen, T. The inclusion-based TOPSIS method with interval-valued intuitionistic fuzzy sets for multiple criteria group decision making. Appl. Soft Comput. 2015, 26, 57-73. [CrossRef] 
36. Pérez-Fernández, R. Monotonicity-based consensus states for the monometric rationalisation of ranking rules with application in decision making. Int. J. Approx. Reason. 2018, 16, 109-110. [CrossRef]

37. Gao, H.; Wei, G.; Huang, Y. Dual Hesitant Bipolar Fuzzy Hamacher Prioritized Aggregation Operators in Multiple Attribute Decision Making. IEEE Access 2018, 6, 11508-11522. [CrossRef]

38. Krishnamurthy, M.; Marcinek, P.; Malik, K.M.; Afzal, M. Representing Social Network Patient Data as Evidence-Based Knowledge to Support Decision Making in Disease Progression for Comorbidities. IEEE Access 2018, 6, 12951-12965. [CrossRef]

39. Rahman, M.A.; Mezhuyev, V.; Bhuiyan, M.Z.A.; Sadat, S.N.; Zakaria, S.A.B.; Refat, N. Reliable Decision Making of Accepting Friend Request on Online Social Networks. IEEE Access 2018, 6, 9484-9491. [CrossRef]

40. Ma, X.; Zhan, J.; Ali, M.I.; Mehmood, N. A survey of decision making methods based on two classes of hybrid soft set models. Artif. Intell. Rev. 2018, 49, 511-529. [CrossRef]

41. Garg, H.; Arora, R. Generalized and group-based generalized intuitionistic fuzzy soft sets with applications in decision-making. Appl. Intell. 2018, 48, 343-356. [CrossRef]

42. Tang, X.Y.; Wei, G.W. Models for green supplier selection in green supply chain management with Pythagorean 2-tuple linguistic information. IEEE Access 2018, 6, 18042-18060. [CrossRef]

43. Wei, G.W.; Lu, M. Pythagorean Fuzzy Maclaurin Symmetric Mean Operators in multiple attribute decision making. Int. J. Intell. Syst. 2018, 33, 1043-1070. [CrossRef]

44. Jiang, F.; Ma, Q. Multi-attribute group decision making under probabilistic hesitant fuzzy environment with application to evaluate the transformation efficiency. Appl. Intell. 2018, 48, 953-965. [CrossRef]

45. Wu, P.; Liu, S.; Zhou, L.; Chen, H. A fuzzy group decision making model with trapezoidal fuzzy preference relations based on compatibility measure and COWGA operator. Appl. Intell. 2018, 48, 46-67. [CrossRef]

46. Kamacı, H.; Atagün, A.O.; Sönmezoğlu, A. Row-products of soft matrices with applications in multiple-disjoint decision making. Appl. Soft Comput. 2018, 62, 892-914. [CrossRef]

47. Wei, G.W.; Gao, H.; Wei, Y. Some q-Rung Orthopair Fuzzy Heronian Mean Operators in Multiple Attribute Decision Making. Int. J. Intell. Syst. 2018. [CrossRef]

48. Wei, G.W.; Lu, M.; Tang, X.Y.; Wei, Y. Pythagorean Hesitant Fuzzy Hamacher Aggregation Operators and Their Application to Multiple Attribute Decision Making. Int. J. Intell. Syst. 2018, 1-37. [CrossRef]

49. Liao, H.C.; Yang, L.Y.; Xu, Z.S. Two new approaches based on ELECTRE II to solve the multiple criteria decision making problems with hesitant fuzzy linguistic term sets. Appl. Soft Comput. 2018, 63, 223-234. [CrossRef]

50. Liu, F.; Liu, Z.-L.; Wu, Y.-H. A group decision making model based on triangular fuzzy additive reciprocal matrices with additive approximation-consistency. Appl. Soft Comput. 2018, 65, 349-359. [CrossRef]

51. Liang, H.; Xiong, W.; Dong, Y. A prospect theory-based method for fusing the individual preference-approval structures in group decision making. Comput. Ind. Eng. 2018, 117, 237-248. [CrossRef]

52. Wu, T.; Liu, X.; Qin, J. A linguistic solution for double large-scale group decision-making in E-commerce. Comput. Ind. Eng. 2018, 116, 97-112. [CrossRef]

53. Wu, H.; Xu, Z.; Ren, P.; Liao, H. Hesitant fuzzy linguistic projection model to multi-criteria decision making for hospital decision support systems. Comput. Ind. Eng. 2018, 115, 449-458. [CrossRef]

54. Xu, Y.; Wen, X.; Zhang, W. A two-stage consensus method for large-scale multi-attribute group decision making with an application to earthquake shelter selection. Comput. Ind. Eng. 2018, 116, 113-129. [CrossRef]

(C) 2018 by the authors. Licensee MDPI, Basel, Switzerland. This article is an open access article distributed under the terms and conditions of the Creative Commons Attribution (CC BY) license (http://creativecommons.org/licenses/by/4.0/). 